\title{
Research Article \\ Relativistic Localizing Processes Bespeak an Inevitable Projective Geometry of Spacetime
}

\author{
Jacques L. Rubin \\ Université de Nice-Sophia Antipolis, Institut de Physique de Nice, UMR7010-UNS-CNRS, Site Sophia Antipolis, 1361 route des Lucioles, \\ 06560 Valbonne, France \\ Correspondence should be addressed to Jacques L. Rubin; jacques.rubin@inphyni.cnrs.fr
}

Received 3 April 2017; Revised 15 June 2017; Accepted 3 July 2017; Published 25 October 2017

Academic Editor: Ralf Hofmann

Copyright (C) 2017 Jacques L. Rubin. This is an open access article distributed under the Creative Commons Attribution License, which permits unrestricted use, distribution, and reproduction in any medium, provided the original work is properly cited. The publication of this article was funded by $\mathrm{SCOAP}^{3}$.

\begin{abstract}
Surprisingly, the issue of events localization in spacetime is poorly understood and a fortiori realized even in the context of Einstein's relativity. Accordingly, a comparison between observational data and theoretical expectations might then be strongly compromised. In the present paper, we give the principles of relativistic localizing systems so as to bypass this issue. Such systems will allow locating users endowed with receivers and, in addition, localizing any spacetime event. These localizing systems are made up of relativistic autolocating positioning subsystems supplemented by an extra satellite. They indicate that spacetime must be supplied everywhere with an unexpected local four-dimensional projective structure besides the well-known three-dimensional relativistic projective one. As a result, the spacetime manifold can be seen as a generalized Cartan space modeled on a four-dimensional real projective space, that is, a spacetime with both a local four-dimensional projective structure and a compatible (pseudo-)Riemannian structure. Localization protocols are presented in detail, while possible applications to astrophysics are also considered.
\end{abstract}

\section{Introduction}

The general principles of the relativistic localizing systems have been defined in a previous paper [1] with just a few details on the projective underlying structure provided by these localizing systems. The latter are based on the so-called relativistic positioning systems [2-9]. The protocols of relativistic positioning are a priori rather simple. For instance, in a four-dimensional spacetime, we can consider four emitting satellites and users with their respective (timelike) worldlines. The four emitters broadcast "emission coordinates" which are no more and no less than time stamps generated by onboard clocks and encoded within EM signals propagating in spacetime. Then, a so-called four-dimensional emission grid can be constructed from this relativistic positioning system and its system of emission coordinates. This grid can be eventually superposed to a grid of reference supplied by a "system of reference" (e.g., the well-known WGS84 system). And then, from this superposition, the positions of the users can be deduced in the given system of reference. More precisely, in relativistic positioning systems, the emitters broadcast not only their own time stamps, but also the time stamps they receive from the others. This process of echoes undergone by the time stamps enables users to construct the fourdimensional emission grid because they can then deduce the spacetime positions of the four emitters. And then, because the positions of the four emitters can be known also in a given system of reference the users can deduce their own positions in this system of reference from their positions in the emission grid.

Here, we focus on the relativistic localizing systems which are systems incorporating relativistic positioning subsystems. We show how causal axiomatics [10-13] and particular projective structures (actually, compasses) homeomorphic to $P \mathbb{R}^{1}$ and $P \mathbb{R}^{2}$ attached all along the worldlines of the emitters of the localizing systems are sufficient to justify a fourdimensional projective structure of the spacetime; in addition to the well-known three-dimensional projective structure.

Beforehand, to proceed in the difficult and delicate description of the relativistic localizing systems, we first need to define as clearly as possible the terminology and the different conventions and notations. 


\section{Notations and Conventions}

We consider a constellation of satellites called "emitters" which typically broadcast numerical values (called "time stamps") generated, for instance, by embarked onboard clocks.

(1) The "main" emitters are denoted by $\mathscr{E}, \overline{\mathscr{E}}, \widetilde{\mathscr{E}}$, and $\widehat{\mathscr{E}}$ with their respective worldlines $\mathscr{W}, \overline{\mathscr{W}}, \widetilde{\mathscr{W}}$, and $\widehat{\mathscr{W}}$. The "ancillary" emitter $\mathcal{S}$ and the "user" $\mathcal{U}$ have their worldlines denoted, respectively, by $\mathscr{W}^{\mathcal{S}}$ and $\mathscr{V}$.

(2) The main emitters constitute the relativistic positioning system.

(3) The ancillary emitter $\mathcal{S}$ and the main emitters constitute the relativistic localizing systems.

(4) The event to be localized is always denoted by the small capital letter $e$.

(5) The user $\mathcal{U}$ collects along its worldline all the data-in particular, the time stamps-from which the localization of the event $e$ is deduced. Among these data, there are those for identifying physically the event $e$ such as, for instance, its shape, its spectrum, and so forth, and which are obtained from apparatus making physical analyses embarked onboard each mean emitter.

(6) Any explicit event will be marked by symbols like “॰," “*," “ ," “, ," and so forth, or also by small capital letters like " $p$," " $\ell$," and so forth. Non-marked or numbered events will refer to general or generic, unspecified events. For instance, $E^{\bullet}$ will be a specified event while $E$ or $E_{2}$ will be generic, unspecified events.

(7) The generic events $E, \bar{E}, \widetilde{E}, \widehat{E}, S$, and $U$ belong, respectively, to the worldlines $\mathscr{W}, \overline{\mathscr{W}}, \widetilde{\mathscr{W}}, \widehat{\mathscr{W}}, \mathscr{W}^{\mathcal{S}}$, and $\mathscr{V}$.

(8) The time stamps will be denoted by the Greek letters " $\tau$," " $\bar{\tau}$," " $\widetilde{\tau}$," “ $\widehat{\tau}$," and “ $\tau$." The first four previous time stamps are "generated" and broadcast, respectively, by the main emitters $\mathscr{E}, \overline{\mathscr{E}}, \widetilde{\mathscr{E}}$, and $\widehat{\mathscr{E}}$, and the last one is "generated" and broadcast by the ancillary emitter $\mathcal{S}$. The four main emitters not only generate their own time stamps but transmit also the time stamps they receive. These main emitters constitute the various autonomous autolocating relativistic positioning systems from which the relativistic localizing systems presented further are constructed.

(9) Two classes of time stamps are considered:

(i) The time stamps which are generated and then broadcast by the emitters at given events on their worldlines. Then, we agree to mark the corresponding time stamps like the given events. For instance, if an emitter generates and broadcasts a time stamp at the specified event $\widetilde{E}^{\bullet}$ or at the generic event $E_{1}$, then the respective time stamps will be denoted by $\tilde{\tau}^{*}$ or $\tau_{1}$.

(ii) The time stamps which are the emission (or time) coordinates of an event $K$-specified or not-will be denoted by “ $\tau_{K}$, , “ $\bar{\tau}_{K}$, " “ $\widetilde{\tau}_{K}$, , “ $\widehat{\tau}_{K}$," and " $\tau_{K}$."

(10) The ancillary emitter $\mathcal{S}$ generates and broadcasts its own time stamp $\dot{\tau}$ and it broadcasts also its time (emission) coordinates provided by the relativistic positioning system. In other words, it is also a particular user of the relativistic positioning system like the user $\mathcal{U}$. Contrarily to the ancillary emitter, the user does not necessarily broadcast its emission coordinates.

(11) Projective frames at events $E$ will be denoted by $\mathfrak{\Im}_{E}$. There are sets of "canonical projective points $[\cdots]_{E}$ " which are the following:

(i) $\mathfrak{\Im}_{E} \equiv\left\{[0]_{E},[1]_{E},[\infty]_{E}\right\}$ for projective frames of the real projective line $P \mathbb{R}^{1}$, and

(ii) $\mathfrak{\mho}_{E} \equiv\left\{[0,0]_{E},[1,1]_{E},[0, \infty]_{E},[\infty, 0]_{E}\right\}$ for projective frames of the 2-dimensional real projective space $P \mathbb{R}^{2}$.

The subscripts will be canceled out if there are no ambiguities on the referring event.

(12) The celestial circles/spheres are denoted by $\mathscr{C}$, and then $\mathscr{C}_{K}$ is the celestial circle/hemisphere at the event $K$. The celestial circles are invoked in the definition of the "echoing systems" of relativistic localizing systems in $(2+1)$-dimensional spacetime presented in Section 4. Considering relativistic localizing systems and their corresponding echoing systems (Section 5$)$ in $(3+1)$-dimensional spacetime, then 2-dimensional projective spaces $P \mathbb{R}^{2}$ are also considered. But, contrarily to the relativistic localizing systems in $(2+1)$-dimensional spacetime, the 2dimensional real projective spaces $P \mathbb{R}^{2}$ cannot be immersed in spheres $S^{2}$ (or $\mathbb{R}^{2}$ ). Then, as well-known from the cell decomposition of $P \mathbb{R}^{2}=\mathbb{R}^{2} \cup P \mathbb{R}^{1}$, the Euclidean space $\mathbb{R}^{2}$ is identified in a standard way with a hemisphere of $S^{2}$ while $P \mathbb{R}^{1}$ is identified with half of the equatorial boundary (see, e.g., [14, p. 10-14] for details).

(13) We denote by (see [15, Def. 3.1, p. R16] and [16]) ${ }^{1}$

(i) “ $\prec$ " the causal order,

(ii) “«” the chronological order, and

(iii) “ $\rightarrow$ ” the horismos (or horismotic relation/order).

(14) We call "emission (or positioning) grid $\mathbb{R}_{P}^{n \text { " the }}$ Euclidean space $\mathbb{R}^{n}$ of positioning, and "localization

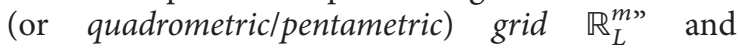
"anisotropic localization (or quadrometric/pentametric) grid $\mathbb{R}_{A L}^{m}$ " two different Euclidean spaces $\mathbb{R}^{m-1} \times \mathbb{R}^{*}$ ascribed to two different, particular sets of time coordinates used for the localization.

(15) The acronyms RPS and RLS mean, respectively, "Relativistic Positioning System" and "Relativistic Localizing System." 


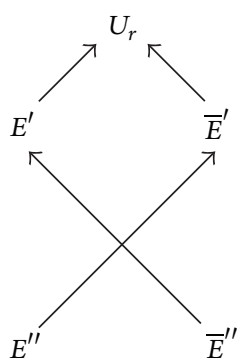

FIGURE 1: The causal structure of the RPS.

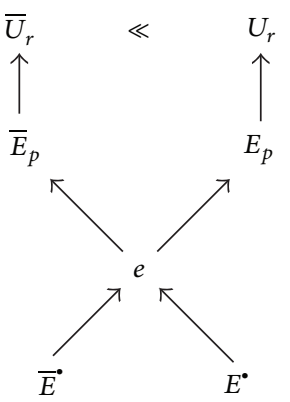

FIgURE 2: The causal structure of the RLS.

\section{RLSs in (1 + 1)-Dimensional Spacetime}

In this $(1+1)$-dimensional case, there are two main emitters $\mathscr{E}$ and $\overline{\mathscr{E}}$ constituting the RPS, and with the ancillary emitter $\mathcal{S}$ they constitute the RLS. We first give the causal structures of the RPS and the associated RLS. In Figures 1 and 2, and also, in all other subsequent figures representing a causal structure, the arrows represent always the horismotic relation between two events.

3.1. The Causal Structure of the RPS. We have the causal structure (see Figure 1 and Table 1) for the autolocating RPS from which the positioning of the user $\mathcal{U}$ is realized.

Then, the position of the user at the event $U_{r}$ in the emission grid $\mathbb{R}_{P}^{2}$ is $U_{r} \equiv\left(\tau^{\prime}, \bar{\tau}^{\prime}\right)$. Also, the user can know from the autolocating process the positions of the two emitters: $E^{\prime} \equiv\left(\tau^{\prime}, \bar{\tau}^{\prime \prime}\right)$ and $\bar{E}^{\prime} \equiv\left(\tau^{\prime \prime}, \bar{\tau}^{\prime}\right)$. Moreover, ephemerides are regularly uploaded onboard the main emitters which broadcast with their time stamps these ephemerides to the users. From these data, that is, ephemerides and positions of the main emitters, the users can deduce their own positions with respect to a given system of reference (e.g., the terrestrial frame of WGS84). This is the core and the important interest of the autolocating positioning systems to immediately furnish the positions of the users with respect to a given system of reference.

3.2. The Causal Structure of the RLS. In this very specific $(1+$ 1)-dimensional case, the localized event $e$ is necessarily the intersection point of two null geodesics. The causal structure is shown in Figure 2 and Table 2.
TABLE 1: The events and their broadcast time stamps in the RPS.

\begin{tabular}{lcc}
\hline Event & Broadcasts time stamp $(\mathrm{s})$ & Received at \\
\hline$E^{\prime \prime}$ & $\tau^{\prime \prime}$ & $\bar{E}^{\prime}$ \\
$\bar{E}^{\prime \prime}$ & $\bar{\tau}^{\prime \prime}$ & $E^{\prime}$ \\
$E^{\prime}$ & $\left(\tau_{E^{\prime}}=\tau^{\prime}, \bar{\tau}^{\prime \prime}\right)$ & $U_{r}$ \\
$\bar{E}^{\prime}$ & $\left(\tau^{\prime \prime}, \bar{\tau}^{\prime}=\bar{\tau}_{\bar{E}^{\prime}}\right)$ & $U_{r}$ \\
\hline
\end{tabular}

TABLE 2: The events and their broadcast time stamps in the RLS.

\begin{tabular}{lcc}
\hline Event & Broadcasts time stamp $(\mathrm{s})$ & Received at \\
\hline$\vec{E}$ & $\bar{\tau}^{*}$ & $E_{p}$ \\
$E^{\cdot}$ & $\tau^{\cdot}$ & $\bar{E}_{p}$ \\
$\bar{E}_{p}$ & $\left(\tau^{\cdot}, \bar{\tau}_{p}=\bar{\tau}_{\bar{E}_{p}}\right)$ & $\bar{U}_{r}$ \\
$E_{p}$ & $\left(\tau_{E_{p}}=\tau_{p}, \bar{\tau}^{\cdot}\right)$ & $U_{r}$ \\
\hline
\end{tabular}

Then, the protocol of localization gives the following time coordinates for $e \equiv\left(\tau_{e}, \bar{\tau}_{e}\right)$ in the localization grid $\mathbb{R}_{L}^{2}: \tau_{e}=$ $\tau_{E^{*}}=\tau^{\bullet}$ and $\bar{\tau}_{e}=\bar{\tau}_{\bar{E}^{*}}=\bar{\tau}^{*}$.

Remark 1. It matters to notice that the two events of reception $U_{r}$ and $\bar{U}_{r}$ are matched by the user on the basis of a crucial identification of the physical data transmitted by the two main emitters (see convention 5) which allow explicitly identifying the physical occurrence of an event $e$. And then, the whole different time stamps collected at these two events can be therefore considered by the user as those needed to make the localization of $e$.

\subsection{Consistency between the Positioning and Localizing Protocols: Identification}

Definition 2 (consistency). We say that the localizing and the positioning protocols or systems are "consistent" if and only if the time coordinates $\left(\tau_{K}, \bar{\tau}_{K}, \ldots\right)$ ascribed to each event $K$ belonging to an emitter's worldline and provided by the localization (resp. positioning) system are the same as those provided by the positioning (resp., localization) system.

Remark 3. In this $(1+1)$-dimensional case, when we identify the time stamps $\tau_{e}$ and $\bar{\tau}_{e}$ with, respectively, $\tau_{E^{*}}$ and $\bar{\tau}_{\bar{E}^{*}}$, then the localization is equivalent to the positioning. This leads to the general Definition 5 below.

Remark 4. The consistency between the localizing and the positioning protocols is not an absolute necessity. We can obtain different time coordinates for the same event $K$ belonging to an emitter's worldline from the positioning system or the localizing one if we change the time stamps ascriptions in the protocols of localization presented further. Then, we can choose arbitrarily the emission grid or the grid of localization to position the event $K$, and, then, we can refer to the preferred grid for the time coordinates ascribed to any other event, positioned or localized. In other words, because the systems of localization include implicitly by construction derived positioning systems, the latter can differ from the initial ones. In this case, the consistency is not satisfied but 


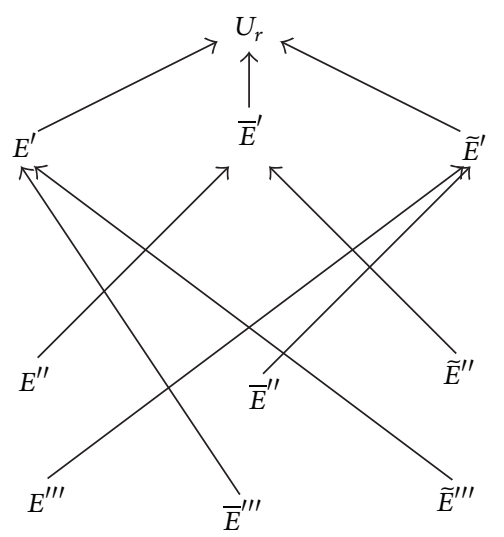

FIgURE 3: The causal structure of the RPS in a $(2+1)$-dimensional spacetime.

we can still refer the time coordinates of any event with respect to the localization grids rather than to the emission grids. The only advantage of the consistency is that once the events are localized then the time coordinates can be ascribed indifferently to any of the two grids.

Definition 5. Let a localized event $e$ and an event $K$ on the worldlines of a main emitter or of the ancillary emitter be such that $e \rightarrow K$ or $K \rightarrow e$ or $e=K$. Then, we call "identification" in the emission (position) grid the ascription of an emission coordinate of $e$ to an emission coordinate of $K$.

\section{RLSs in $(2+1)$-Dimensional Spacetime}

In this case, there are three main emitters $\mathscr{E}, \overline{\mathscr{E}}$, and $\widetilde{\mathscr{E}}$ constituting the autolocating RPS and, again, an ancillary emitter $\mathcal{S}$ with which they constitute the RLS.

4.1. The Causal Structure of the RPS. This causal structure is described in Figure 3 and Table 3.

Then, the position in the emission grid $\mathbb{R}_{P}^{3}$ of the user at $U_{r}$ is $\left(\tau^{\prime}, \bar{\tau}^{\prime}, \tilde{\tau}^{\prime}\right)$, and those of $E^{\prime}, \bar{E}^{\prime}$, and $\widetilde{E}^{\prime}$ are, respectively, $\left(\tau^{\prime}, \bar{\tau}^{\prime \prime \prime}, \widetilde{\tau}^{\prime \prime \prime}\right),\left(\tau^{\prime \prime}, \bar{\tau}^{\prime}, \widetilde{\tau}^{\prime \prime}\right)$, and $\left(\tau^{\prime \prime \prime}, \bar{\tau}^{\prime \prime}, \widetilde{\tau}^{\prime}\right)$.

Remark 6. It matters to notice that in autolocating RPSs the time stamp broadcast by each main emitter is also one of its emission coordinates, for example, $\tau_{E^{\prime}}=\tau^{\prime}$ for $\mathscr{E}$ at $E^{\prime}$ in Table 1 and $\bar{\tau}^{\prime}=\bar{\tau}_{\bar{E}^{\prime}}$ for $\overline{\mathscr{E}}$ at $\bar{E}^{\prime}$ in Table 3 . This property is common to any RPS whatever is the spacetime dimension.

\subsection{The Description of the RLS and Its Causal Structures.} The determination of the first emission coordinate $\tau_{e}$ for the event $e$ to be localized is obtained from a first system of light "echoes" associated with the privileged emitter $\mathscr{E}$. And then, this system is linked to one event of reception $U_{r} \in \mathscr{V}$ where all the time stamps are collected by the user. We denote by $\mathscr{E} \mathrm{ch}$ this system of light "echoes" on the worldline of the given, privileged emitter $\mathscr{E}$.

Also, one of the key ingredient in the echoing process presented below is the way any event $K$ in the past null cone
TABLE 3: The events and their broadcast time stamps.

\begin{tabular}{lcc}
\hline Event & Broadcasts time stamp(s) & Received at \\
\hline$E^{\prime \prime \prime}$ & $\tau^{\prime \prime \prime}$ & $\widetilde{E}^{\prime}$ \\
$\bar{E}^{\prime \prime \prime}$ & $\bar{\tau}^{\prime \prime \prime}$ & $E^{\prime}$ \\
$\widetilde{E}^{\prime \prime \prime}$ & $\widetilde{\tau}^{\prime \prime \prime}$ & $E^{\prime}$ \\
$E^{\prime \prime}$ & $\tau^{\prime \prime}$ & $\bar{E}^{\prime}$ \\
$\bar{E}^{\prime \prime}$ & $\bar{\tau}^{\prime \prime}$ & $\widetilde{E}^{\prime}$ \\
$\widetilde{E}^{\prime \prime}$ & $\widetilde{\tau}^{\prime \prime}$ & $\bar{E}^{\prime}$ \\
$E^{\prime}$ & $\left(\tau_{E^{\prime}}=\tau^{\prime}, \bar{\tau}^{\prime \prime \prime}, \widetilde{\tau}^{\prime \prime \prime}\right)$ & $U_{r}$ \\
$\bar{E}^{\prime}$ & $\left(\tau^{\prime \prime}, \bar{\tau}^{\prime}=\bar{\tau}_{\bar{E}^{\prime}}, \widetilde{\tau}^{\prime \prime}\right)$ & $U_{r}$ \\
$\widetilde{E}^{\prime}$ & $\left(\tau^{\prime \prime \prime}, \bar{\tau}^{\prime \prime}, \widetilde{\tau}_{\vec{E}}=\widetilde{\tau}^{\prime}\right)$ & $U_{r}$ \\
\hline
\end{tabular}

of $E_{p}$ is associated with a "bright" point on the celestial circle $\mathscr{C}_{E_{p}}$ (see Figure 8 ). Because $K \rightarrow E_{p}$, we can only consider null "directions" $k_{E_{p}}$ at the origin $E_{p}$ and tangent at $E_{p}$ to the null geodesic joining $K$ to $E_{p}$. The abstract space whose element are these past null directions we call $\mathscr{N}^{-}$. This space can be represented by the intersection $\mathscr{C}_{E_{p}}$ of the past null cone with a space-like surface passing through an event $N_{p} \in$ $\mathscr{W}$ in the past vicinity of $E_{p}$, that is, $N_{p} \ll E_{p}$. Then, the exterior of this celestial circle represents space-like directions.

In physical terms, the significance of $\mathscr{C}_{E_{p}}$ is the following. Light rays reaching the event $E_{p}$ and detected by the "eye" of the satellite correspond to null lines through $E_{p}$ whose past directions constitutes the field of vision of the "observing" satellite. This is $\mathcal{N}^{-}$and it is represented by the celestial circle $\mathscr{C}_{E_{p}}$ which is an accurate geometrical representation of what the satellite actually "sees." For, the satellite can be considered as permanently situated at the center of a unit circle (his circle of vision) onto which the satellite maps all it detects at any instant. Then, the mapping of the past null directions at $E_{p}$ to the points of $\mathscr{C}_{E_{p}}$ we can call the sky mapping. Additionally, because (1) the circle $S^{1}$ is homeomorphic to the real projective line $P \mathbb{R}^{1}$ and (2) we need angle measurements to frame the points of $\mathscr{C}_{E_{p}}$ associated with any event $K$ in the past null cone of $E_{p}$ to be furthermore localized, then a particular production process of projective frame for $\mathscr{C}_{E_{p}}$ must be devised and incorporated in the echoing system definition now given below.

Definition 7 (the echoing system $\mathscr{E} \mathrm{ch}$ ). The echoing system $\mathscr{E}$ ch associated with the privileged emitter $\mathscr{E}$ is based on the following features (see Figure 4 and Table 4):

(i) One primary event $E_{p}$ with its celestial circle $\mathscr{C}_{E_{p}}$.

(ii) Three secondary events $\bar{E}^{\bullet}, \widetilde{E}^{\bullet}$, and $\mathcal{S}^{\bullet}$, associated, respectively, with the canonical projective points $[0]_{E_{p}},[\infty]_{E_{p}}$, and $[1]_{E_{p}}$ of the projective frame $\mathfrak{F}_{E_{p}}$ defined on $\mathscr{C}_{E_{p}}$.

(iii) Two ternary events: $E^{\diamond}$ and $E^{\prime \prime}$.

(iv) A compass on $\mathscr{C}_{E_{p}}$ with a moving origin anchored on the projective point $[1]_{E_{p}}$ of $\mathscr{C}_{E_{p}}$ associated with $\mathcal{S}^{\bullet}$. 


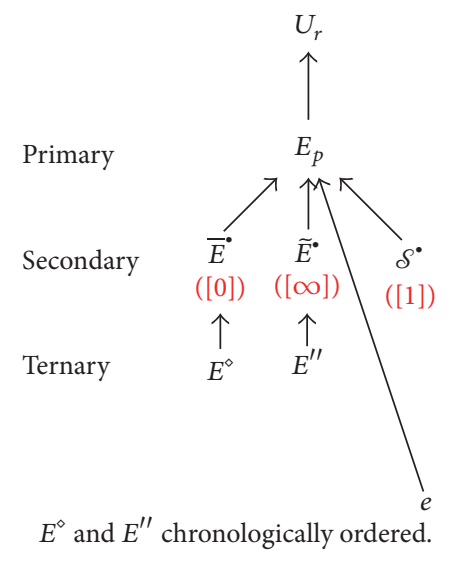

FIgURE 4: The causal structure of $\mathscr{E}$ ch with the event $e$.

TABle 4: The events and their broadcast time stamps in the $\mathscr{E} \mathrm{ch}$ system.

\begin{tabular}{lcc}
\hline Event & Broadcasts time stamp & Received at \\
\hline$E^{\diamond}$ & $\tau^{\diamond}$ & $\vec{E}$ \\
$E^{\prime \prime}$ & $\tau^{\prime \prime}$ & $\widetilde{E}$ \\
$\mathcal{S}^{\bullet}$ & $\tau_{\delta^{\bullet}}$ & $E_{p}$ \\
$\vec{E}$ & $\tau^{\diamond}$ & $E_{p}$ \\
$\widetilde{E}^{\cdot}$ & $\tau^{\prime \prime}$ & $E_{p}$ \\
\hline
\end{tabular}

(v) An event of reception $U_{r} \in \mathscr{V}$ at which all the data are collected and sent by the emitter $\mathscr{E}$.

The determination of the second (resp., third) emission coordinate $\bar{\tau}_{e}$ (resp., $\widetilde{\tau}_{e}$ ) for the event $e$ to be localized is obtained from a second (resp., third) system of "echoes" associated with the privileged emitter $\overline{\mathscr{E}}$ (resp., $\widetilde{\mathscr{E}}$ ). It is also linked to one event of reception $\bar{U}_{\ell}$ (resp. $\widetilde{U}_{\ell}$ ) where all the time stamps are collected. We denote by $\overline{\mathscr{E}} \mathrm{ch}$ (resp., $\widetilde{\mathscr{E}} \mathrm{ch}$ ) this second (resp., third) system of "echoes" on the worldline of the privileged emitter $\overline{\mathscr{E}} \mathrm{ch}$ (resp., $\widetilde{\mathscr{E}} \mathrm{ch}$ ).

Then, we have the following.

Definition 8 (the echoing systems $\overline{\mathscr{E}} \mathrm{ch}$ and $\widetilde{\mathscr{E}} \mathrm{ch}$ ). The definitions of the echoing systems $\overline{\mathscr{E}}$ ch and $\widetilde{\mathscr{E}}$ ch are obtained when making the following substitutions of events and marks in the definition of $\mathscr{E} \mathrm{ch}$ :

(i) For $\overline{\mathscr{E}} \mathrm{ch}:(U, E, \bar{E}, \widetilde{E}) \rightarrow(\bar{U}, \bar{E}, \widetilde{E}, E)$ and $\bullet \rightarrow *$,

(ii) For $\widetilde{\mathscr{E}} \mathrm{ch}:(U, E, \bar{E}, \widetilde{E}) \rightarrow(\widetilde{U}, \widetilde{E}, E, \bar{E})$ and $\bullet \rightarrow$ '.

Then, we have the causal structure of the echoing system $\mathscr{E}$ ch (Figure 4 and Table 4); the other two causal structures for $\overline{\mathscr{E}} \mathrm{ch}$ and $\widetilde{\mathscr{E}} \mathrm{ch}$ (Figures 5 and 6 ) are deduced from the causal structure of $\mathscr{E} \mathrm{ch}$ by making the substitutions indicated in Definition 8. We indicate also the three structures with the event $e$ (Figure 7).

Remark 9. Again (Remark 1), it matters to notice that the three events of reception $U_{r}, \bar{U}_{r}$, and $\widetilde{U}_{r}$ (Figure 7) are matched by the user on the basis of an identification of the

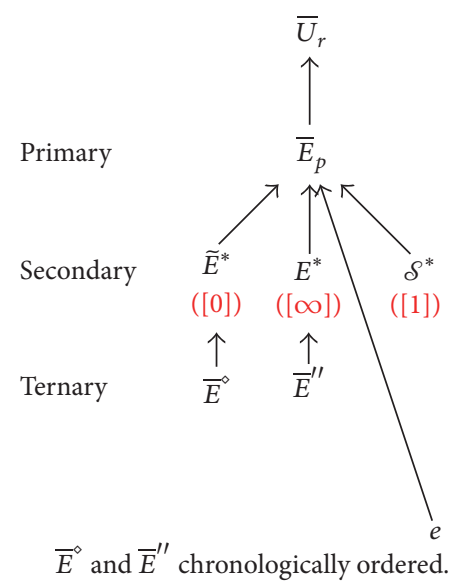

FIGURE 5: The causal structure of $\overline{\mathscr{E}}$ ch with the event $e$.

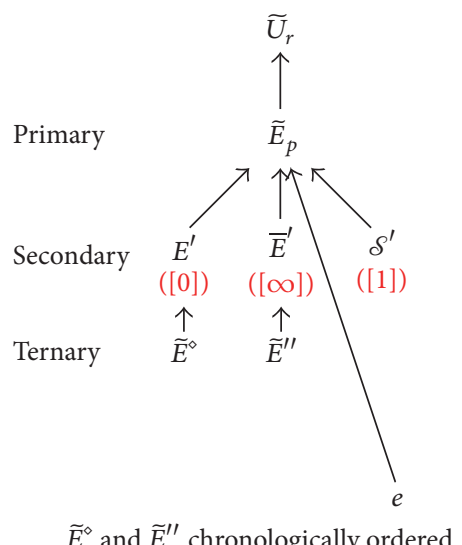

FiguRE 6: The causal structure of $\widetilde{\mathscr{E}} \mathrm{ch}$ with the event $e$.

physical data for $e$ transmitted by the main emitters (see convention 5).

4.3. The Projective Frames and the Time Stamps Correspondences. The realization of the RLS is based on a sort of spacetime parallax, that is, a passage from angles " $\alpha$ " measured on celestial circles to spatiotemporal distances. And thus, because spatiotemporal distances are evaluated from time stamps " $\tau$ " in the present context, we need to make the translation of angles into time stamps. This involves onboard compasses embarked on each main emitter to find somehow the bearings. Then, this translation is neither more nor less than a change of projective frames.

To make this change of projective frames effective, we need to define the projective frames on the celestial circles attached to each main emitter. This can be done ascribing to specific "bright points" detected on the celestial circles both angles and time stamps. This ascription is then naturally achieved if these bright points are the main emitters themselves since they broadcast the time stamps. But, if we have three emitters for the RPS, then only two bright points can be detected on each celestial circle attached to each main emitter. And, we need three bright points to have a projective frame 


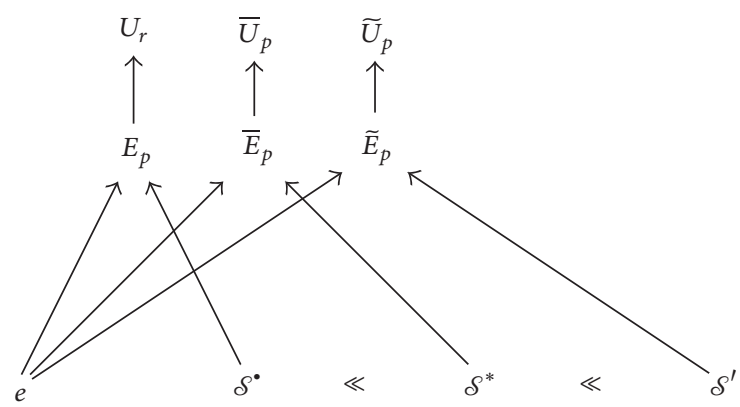

FIgURE 7: The causal structure for the three echoing systems $\mathscr{E} \mathrm{ch}$, $\overline{\mathscr{E}} \mathrm{ch}$, and $\widetilde{\mathscr{E}} \mathrm{ch}$ with the event $e$. The chronological order between $\mathcal{S}^{*}, \mathcal{S}^{*}$, and $\mathcal{S}^{\prime}$ belonging to $\mathscr{W}^{\mathcal{S}}$ can be different.

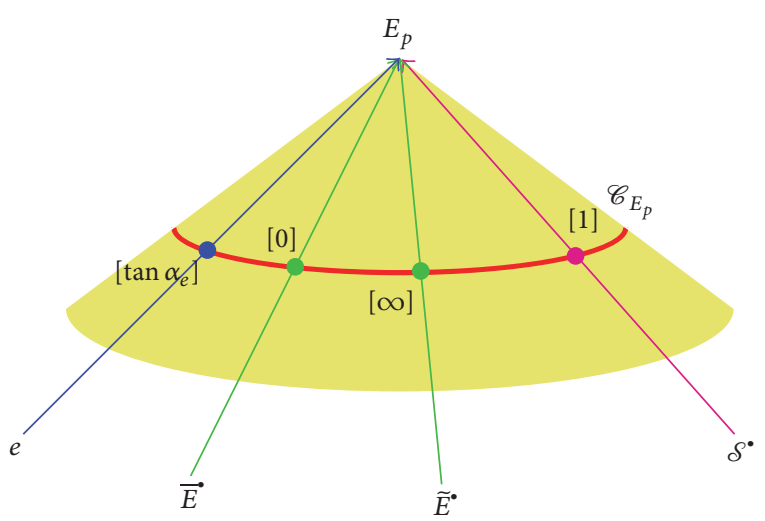

FIGURE 8: The past null cone and the celestial circle $\mathscr{C}_{E_{p}}$.

TABLE 5: The change of projective frame and the corresponding events.

\begin{tabular}{lcc}
\hline Event & $\mathfrak{\mho}_{E_{p}}$ & $\mathfrak{F}_{E_{p}}^{\tau}$ \\
\hline$e$ & {$\left[\tan \alpha_{e}\right]$} & {$\left[\tau_{e}\right]$} \\
$\vec{E}$ & {$[0]$} & {$\left[\tau^{\diamond}\right]$} \\
$\vec{E}$ & {$[\infty]$} & {$\left[\tau^{\prime \prime}\right]$} \\
$\mathcal{S}^{*}$ & {$[1]$} & {$\left[\tau_{\delta^{*}}\right]$} \\
\hline
\end{tabular}

on the celestial circle homeomorphic to $P \mathbb{R}^{1}$, hence the need for the ancillary emitter $\delta$. The change of projective frames is described in Table 5 and Figures 8 and 9. For instance, the main emitter $\overline{\mathscr{E}}$ broadcasts the time stamp $\tau^{\diamond}$ at the secondary event $\vec{E}$, and the former is then received by the emitter $\mathscr{E}$ at the primary event $E_{p}$. Also, if $\overline{\mathscr{E}}$ is always associated by convention with the canonical projective point $[0]_{E_{p}}$ on the celestial circle of $\mathscr{E}$, then we deduce that $\tau^{\diamond}$ corresponds by a projective transformation to 0 . And then, we proceed in the same way with the other two canonical projective points.
As a result, the relations between the angles and the time stamps are the following:

$$
\begin{aligned}
\tan \alpha_{e} & =\left[\widetilde{\tau}^{\diamond}: \tau^{\prime \prime} \mid \tau_{e}: \tau_{\delta^{\bullet}}\right]=\frac{\left[\tau^{\diamond}: \tau^{\prime \prime} \mid \tau_{e}\right]}{\left[\tau^{\diamond}: \tau^{\prime \prime} \mid \tau_{\delta^{\bullet}}\right]} \\
& \equiv \mathfrak{M}\left(\tau_{e}\right), \\
\tan \bar{\alpha}_{e} & =\left[\bar{\tau}^{\diamond}: \bar{\tau}^{\prime \prime} \mid \bar{\tau}_{e}: \bar{\tau}_{\delta^{*}}\right]=\frac{\left[\bar{\tau}^{\diamond}: \bar{\tau}^{\prime \prime} \mid \bar{\tau}_{e}\right]}{\left[\bar{\tau}^{\diamond}: \bar{\tau}^{\prime \prime} \mid \bar{\tau}_{\delta^{*}}\right]} \\
& \equiv \overline{\mathfrak{M}}\left(\bar{\tau}_{e}\right), \\
\tan \widetilde{\alpha}_{e} & =\left[\widetilde{\tau}^{\diamond}: \widetilde{\tau}^{\prime \prime} \mid \widetilde{\tau}_{e}: \widetilde{\tau}_{\delta^{\prime}}\right]=\frac{\left[\widetilde{\tau}^{\diamond}: \tilde{\tau}^{\prime \prime} \mid \tilde{\tau}_{e}\right]}{\left[\widetilde{\tau}^{\diamond}: \widetilde{\tau}^{\prime \prime} \mid \widetilde{\tau}_{\delta^{\prime}}\right]} \\
& \equiv \widetilde{\mathfrak{M}}\left(\widetilde{\tau}_{e}\right),
\end{aligned}
$$

where $[a: b \mid c: d]$ is the cross-ratio of the four projective points $a, b, c$, and $d$ :

$$
\begin{aligned}
& {[a: b \mid c: d]=\frac{[a: b \mid c]}{[a: b \mid d]}} \\
& \quad \text { where }[a: b \mid c]=[a: b \mid c: \infty]=\left(\frac{a-c}{b-c}\right) .
\end{aligned}
$$

Conversely, the time coordinates for the event $e$ are then obtained from the angles measurements and the following formulas:

$$
\begin{aligned}
\tau_{e} & =\left(\frac{\tau^{\diamond}-\tau^{\prime \prime}\left[\tau^{\diamond}: \tau^{\prime \prime} \mid \tau_{\delta^{*}}\right] \tan \alpha_{e}}{1-\left[\tau^{\diamond}: \tau^{\prime \prime} \mid \tau_{\delta^{*}}\right] \tan \alpha_{e}}\right), \\
\bar{\tau}_{e} & =\left(\frac{\bar{\tau}^{\diamond}-\bar{\tau}^{\prime \prime}\left[\bar{\tau}^{\diamond}: \bar{\tau}^{\prime \prime} \mid \bar{\tau}_{\delta^{*}}\right] \tan \bar{\alpha}_{e}}{1-\left[\bar{\tau}^{\diamond}: \bar{\tau}^{\prime \prime} \mid \bar{\tau}_{\delta^{*}}\right] \tan \bar{\alpha}_{e}}\right), \\
\tilde{\tau}_{e} & =\left(\frac{\widetilde{\tau}^{\diamond}-\widetilde{\tau}^{\prime \prime}\left[\widetilde{\tau}^{\diamond}: \tilde{\tau}^{\prime \prime} \mid \tilde{\tau}_{\delta^{\prime}}\right] \tan \widetilde{\alpha}_{e}}{1-\left[\widetilde{\tau}^{\diamond}: \tilde{\tau}^{\prime \prime} \mid \tilde{\tau}_{\delta^{\prime}}\right] \tan \tilde{\alpha}_{e}}\right) .
\end{aligned}
$$

And thus, the event $e$ is localized in the localization grid $\mathbb{R}_{L}^{3}$. Then, we deduce the following lemma.

Lemma 10. The map

$$
\mathbb{S}:\left(\tan \alpha_{e}, \tan \bar{\alpha}_{e}, \tan \widetilde{\alpha}_{e}\right) \in \mathscr{T}^{3} \longrightarrow\left(\tau_{e}, \bar{\tau}_{e}, \widetilde{\tau}_{e}\right) \in \mathscr{T}^{3},
$$

where $\mathscr{T}^{3} \equiv\left(P \mathbb{R}^{1}\right)^{3}$ is an automorphism.

Proof. This is obvious from the relations (1a)-(1c), because $\mathfrak{M}, \overline{\mathfrak{M}}$, and $\widetilde{\mathfrak{M}}$ are bijective Möbius transformations.

\subsection{The Consistency between the Positioning and Localization Protocols}

Theorem 11. The localization and the positioning protocols or systems in a $(2+1)$-dimensional spacetime are consistent. 


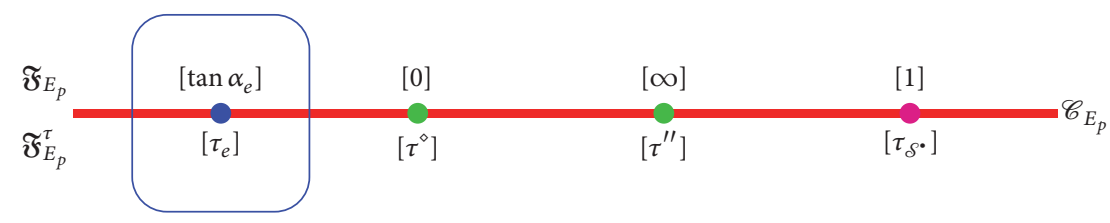

FIGURE 9: The projective line associated with the celestial circle $\mathscr{C}_{E_{p}}$.

Proof. The consistency must be satisfied if $e$ is an element of the emitters' worldlines. Indeed, the localization protocol is consistent with the positioning protocol if the set of events on the emitters' worldlines from which the localization of any event $e$ is possible are themselves localizable.

Case $1\left(e \in \mathscr{W}^{\mathcal{\delta}}\right)$. We consider two cases: $e \ll \mathcal{S}^{\bullet}$ and $\mathcal{S}^{\bullet} \ll e$. The other cases with $\mathcal{S}^{*}$ or $S^{\prime}$ instead of $\mathcal{S}^{\bullet}$ give the same results. Now, we start with the assumption $e \ll \mathcal{S}^{\bullet}$ from which we deduce the following causal structure:

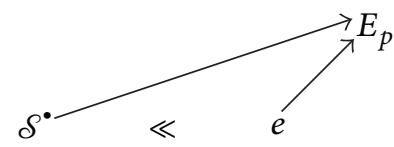

In particular, from $\mathcal{S}^{\bullet} \ll e \rightarrow E_{p}$, we find ${ }^{2}$ that $\mathcal{S}^{\bullet} \prec$ $e \prec E_{p}$, and then, with $\mathcal{S}^{\bullet} \rightarrow E_{p}$, we obtain ${ }^{3} \mathcal{S}^{\bullet} \rightarrow e \rightarrow E_{p}$. But then ${ }^{2}$, we have $\mathcal{S}^{\bullet} \ll e$. With the assumption $e \ll \mathcal{S}^{\bullet}$, we deduce also $e \ll \mathcal{S}^{\circ}$, and therefore $\mathcal{S}^{\bullet}=e$. Hence, we consider that $\mathcal{S}^{\bullet}=e$ and with the other two sets of events $\mathcal{S}^{*}$ with $\bar{E}_{p}$ or $S^{\prime}$ and $\widetilde{E}_{p}$, we deduce finally that $e=\mathcal{S}^{\bullet}=\mathcal{S}^{*}=$ $S^{\prime} \equiv S$. Therefore, we conclude that the time coordinates of $e$ provided by the positioning system are $\tau_{e}=\tau_{S}, \bar{\tau}_{e}=\bar{\tau}_{S}$, and $\tilde{\tau}_{e}=\tilde{\tau}_{S}$.

Besides, from the projective frames, we have also

$$
\begin{aligned}
& \tan \alpha_{e}=\left[\tau^{\diamond}: \tau^{\prime \prime} \mid \tau_{e}: \tau_{\delta^{*}}\right]=1, \\
& \tan \bar{\alpha}_{e}=\left[\bar{\tau}^{\diamond}: \bar{\tau}^{\prime \prime} \mid \bar{\tau}_{e}: \bar{\tau}_{\delta^{*}}\right]=1, \\
& \tan \widetilde{\alpha}_{e}=\left[\widetilde{\tau}^{\diamond}: \widetilde{\tau}^{\prime \prime} \mid \tilde{\tau}_{e}: \tilde{\tau}_{\delta^{\prime}}\right]=1 .
\end{aligned}
$$

And therefore, we obtain

$$
\begin{aligned}
& \tau_{e}=\tau_{\mathcal{S}^{*}}, \\
& \bar{\tau}_{e}=\bar{\tau}_{\mathcal{S}^{*}}, \\
& \widetilde{\tau}_{e}=\tilde{\tau}_{\mathcal{S}^{\prime}},
\end{aligned}
$$

which are the coordinates of $S$.

In conclusion, the localization protocol is consistent with the positioning one.

Case 2 ( $e$ is a primary event: $e=E_{p}$ (primary) $\in \mathscr{W}$ ). In this case, we obtain the causal structure (Figure 10).

Then, from the three echoing causal structures $\mathscr{E} \mathrm{ch}, \overline{\mathscr{E}} \mathrm{ch}$, and $\widetilde{\mathscr{E}} \mathrm{ch}$, we have $e=E_{p}=E^{*}=E^{\prime}$ where $E^{*}$ is associated with the projective point $[\infty]_{\bar{E}_{p}}$ and $E^{\prime}$ is associated with the projective point $[0]_{\tilde{E}_{p}}$. Consequently, we have $\tan \bar{\alpha}_{e}=\infty$ and $\tan \widetilde{\alpha}_{e}=0$. Also, we have $\vec{E}=\bar{E}^{\prime \prime}$ and $\widetilde{E}^{\bullet}=\widetilde{E}^{\diamond}$ from which we deduce from the positioning system that their time coordinates are equal; that is, we have (one of the emission coordinates is equal to the broadcast one in the positioning protocol; see Remark 6)

$$
\begin{aligned}
& \bar{\tau}_{\vec{E}^{*}}=\bar{\tau}^{\bullet}=\bar{\tau}^{\prime \prime}=\bar{\tau}_{\bar{E}^{\prime \prime}}, \\
& \widetilde{\tau}_{\vec{E}}=\widetilde{\tau}^{\bullet}=\widetilde{\tau}^{\diamond}=\tilde{\tau}_{\vec{E}} .
\end{aligned}
$$

Besides, from the localization protocol, we have

$$
\begin{aligned}
& \tan \alpha_{e}=\left[\tau^{\diamond}: \tau^{\prime \prime} \mid \tau_{e}: \tau_{\mathcal{S}^{*}}\right]=? \text { (not defined) }, \\
& \tan \bar{\alpha}_{e}=\left[\bar{\tau}^{\diamond}: \bar{\tau}^{\prime \prime} \mid \bar{\tau}_{e}: \bar{\tau}_{\mathcal{S}^{*}}\right]=\infty, \\
& \tan \widetilde{\alpha}_{e}=\left[\widetilde{\tau}^{\diamond}: \tilde{\tau}^{\prime \prime} \mid \tilde{\tau}_{e}: \tilde{\tau}_{\mathcal{S}^{\prime}}\right]=0 .
\end{aligned}
$$

Hence, we deduce

$$
\begin{aligned}
& \tau_{e}=?, \\
& \bar{\tau}_{e}=\bar{\tau}^{\prime \prime}, \\
& \tilde{\tau}_{e}=\tilde{\tau}^{\diamond} .
\end{aligned}
$$

And from the positioning protocol, because $E_{p}$ is a positioned point with emission coordinates $\left(\tau_{E_{p}}, \vec{\tau}^{*}, \widetilde{\tau}^{\bullet}\right)$, we have also

$$
\begin{aligned}
& \bar{\tau}_{e}=\bar{\tau}_{E_{p}}=\bar{\tau}^{\cdot}, \\
& \widetilde{\tau}_{e}=\widetilde{\tau}_{E_{p}}=\tilde{\tau}^{\cdot},
\end{aligned}
$$

and therefore, with (8), we deduce the consistency for two time stamps. Actually, $\tau_{e}$ is not obtained by localization but by identification (Definition 5). Indeed, we know that $e$ is an element of $\mathscr{W}$ and that $\tau_{e}=\tau_{E_{p}}$ is broadcast by the identified main emitter $\mathscr{E}$. This determination of $\tau_{e}$ is then similar to the emission coordinate ascription presented in the $(1+1)$-dimensional case for which localization is equivalent to positioning (Remark 3), hence the consistency.

Case 3 ( $e$ is a secondary event: $e=\bar{E}$ (secondary) $\in \overline{\mathscr{W}}$ or $e=\bar{E}^{\prime}$ (secondary) $\in \overline{\mathscr{W}}$ ). Then, the causal structure is the following whenever $e=\bar{E}^{\bullet}$ (Figure 11):

(i) $e=\bar{E}^{\bullet}$ (secondary) $\in \overline{\mathscr{W}}$. Then, the localization protocol at $E_{p}$ gives the formula $\tan \alpha_{e}=\left[\tau^{\diamond}: \tau^{\prime \prime} \mid \tau_{e}\right.$ : 


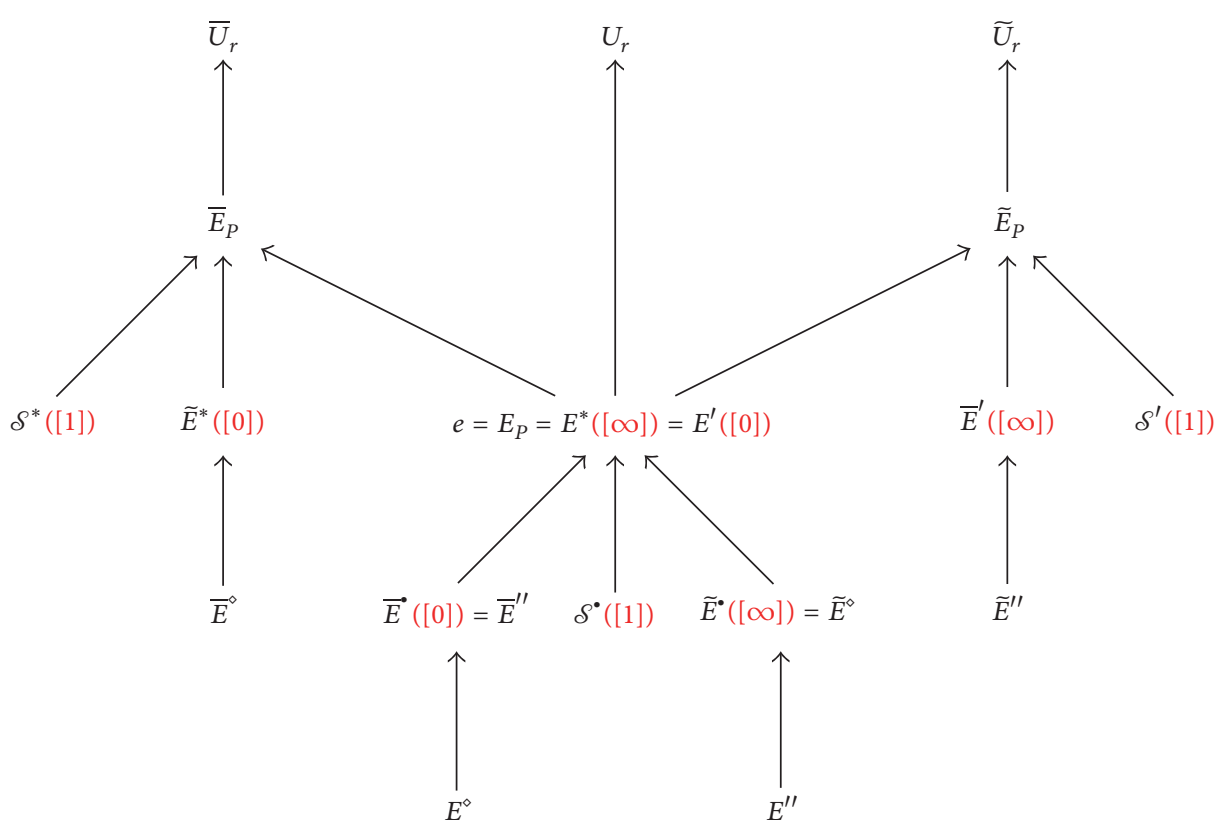

FIGURE 10: The causal structure for the three echoing protocols $\mathscr{E} \mathrm{ch}, \overline{\mathscr{E}} \mathrm{ch}$, and $\widetilde{\mathscr{E}} \mathrm{ch}$ whenever $e=E_{P}$.

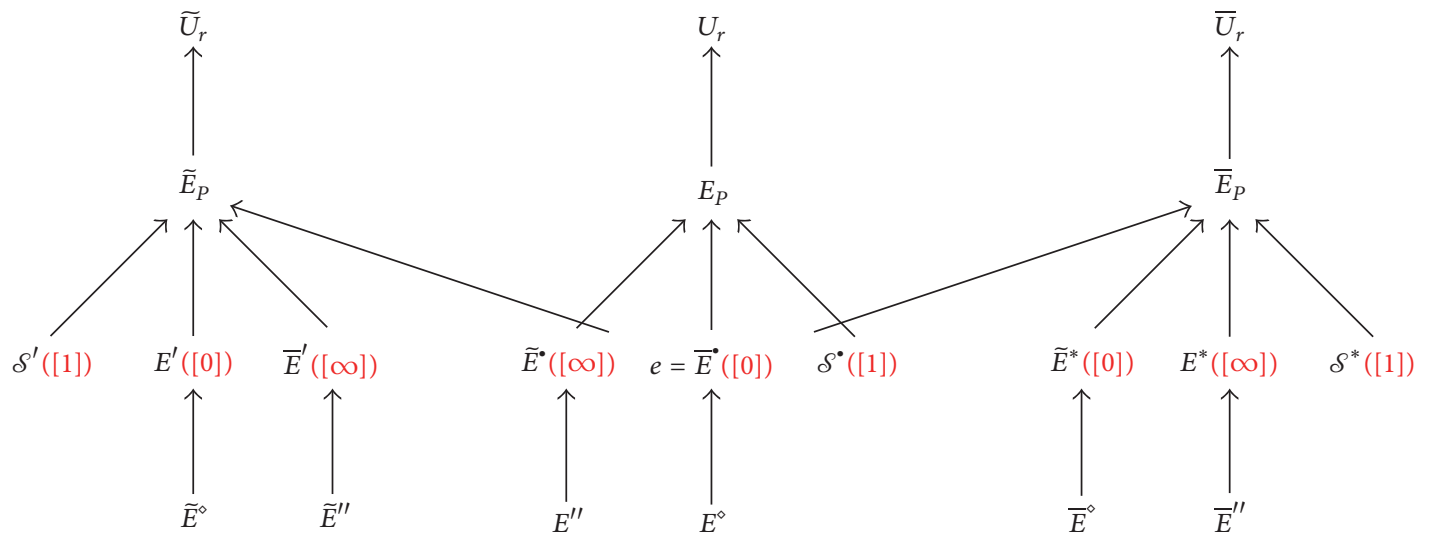

FIGURE 11: The causal structure for the three echoing protocols $\mathscr{E} \mathrm{ch}, \overline{\mathscr{E}} \mathrm{ch}$, and $\widetilde{\mathscr{E}} \mathrm{ch}$ whenever $e=\vec{E}$.

$\left.\tau_{s^{*}}.\right]=0$ because $\bar{E}^{*}$ is associated with the projective point $[0]_{E_{p}}$. Therefore, we have $\tau_{e}=\tau^{\diamond}$. But, from the positioning protocol, the emission coordinate $\tau_{\vec{E}}$. of $\bar{E}^{\bullet}$ relative to the main emitter $\mathscr{E}$ is $\tau^{\diamond}$ broadcast at the ternary event $E^{\diamond}$. Hence, $\tau_{\vec{E}} \cdot=\tau^{\diamond}$ and we deduce the consistency of the localization protocol with the positioning protocol for one emission coordinate.

(ii) $e=\bar{E}^{\prime}$ (secondary) $\in \overline{\mathscr{W}}$. The reasoning is similar to the previous one. Then, we deduce the consistency for $\widetilde{\tau}_{e}=\widetilde{\tau}_{\bar{E}^{\prime}}=\widetilde{\tau}^{\prime \prime}$ because (1) $\widetilde{E}_{p}$ is the primary event for $\bar{E}^{\prime}$ and $\widetilde{E}^{\prime \prime}$ is the ternary event for $\bar{E}^{\prime}$, and (2) $\left[\widetilde{\tau}^{\diamond}\right.$ : $\left.\widetilde{\tau}^{\prime \prime} \mid \widetilde{\tau}_{e}: \widetilde{\tau}_{\delta^{\prime}}\right]=\infty$ which involves $\widetilde{\tau}_{e}=\widetilde{\tau}^{\prime \prime}$.
Now, we consider two distinct causal structures of localization $a$ and $b$ such that $e=\bar{E}^{\bullet a}=\bar{E}^{\prime b}$ from which we deduce the consistency for $\tau_{e}$ and $\widetilde{\tau}_{e}$. Furthermore, as in Case 2, we deduce $\bar{\tau}_{e}$ by identification (Definition 5) and we obtain $\bar{\tau}_{e}=$ $\bar{\tau}_{\bar{E}^{a}}=\bar{\tau}_{\bar{E}^{b}}$, hence the consistency.

Case 4 ( $e$ is a ternary event). For instance, we can set $e=E^{\diamond}$. But then, we have also $e=E^{\diamond} \rightarrow E_{P}$ on $\mathscr{W}^{\mathscr{C}}$ which is impossible since we have only the chronological order on the emitters' worldlines.

Remark 12. From this theorem, we can then notice that RLSs are based on autolocalization protocols similarly to RPSs which are based on autolocation protocols. As a result, RLSs and RPSs are independent of any system of reference. 


\subsection{The Local Projective Structure}

Definition 13. We call

(i) Emission grid the Euclidean space $\mathbb{R}_{P}^{3} \equiv \mathbb{R}^{3}$ of the positioned events $e_{P}=\left(\tau_{e}, \bar{\tau}_{e}, \widetilde{\tau}_{e}\right)$;

(ii) Localization (or quadrometric) grid the Euclidean space $\mathbb{R}_{L}^{4} \equiv \mathbb{R}^{3} \times \mathbb{R}^{*}$ of the localized events $e_{L}=$ $\left(\tau_{e}, \bar{\tau}_{e}, \widetilde{\tau}_{e}, \dot{\tau}_{e}\right)$, where $\dot{\tau}_{e}$ is provided by the ancillary emitter $\delta$ by identification from the horismotic relation $S^{L} \rightarrow e_{P}\left(S^{L} \in \mathscr{W}^{\mathcal{S}}\right)$ or the "message function" [11] $f_{\mathcal{S}}^{-}: \mathbb{R}_{P}^{3} \rightarrow \mathscr{W}^{\mathcal{S}}$; that is, the time stamp $\stackrel{\circ}{\tau}^{L}$ broadcast by $\mathcal{S}$ at $S^{L}$ is such that ${ }^{L}{ }^{L} \equiv \dot{\tau}_{e}$;

(iii) Anisotropic localization (or quadrometric) grid the Euclidean space $\mathbb{R}_{A L}^{4} \equiv \mathbb{R}^{3} \times \mathbb{R}^{*}$ of events $e_{A L}=$ $\left(\dot{\tau}_{e} \tau_{e}, \dot{\tau}_{e} \bar{\tau}_{e}, \dot{\tau}_{e} \widetilde{\tau}_{e}, \dot{\tau}_{e}\right)$.

Definition 14. We denote by $I: \mathbb{R}_{L}^{4} \rightarrow \mathbb{R}_{A L}^{4}$ the bijective map such that $I\left(e_{L}\right)=e_{A L}$. And we denote by $\pi: \mathbb{R}_{A L}^{4} \rightarrow \mathbb{R}_{P}^{3}$ the submersion such that $\pi\left(e_{A L}\right)=e_{P}$.

Remark 15. In these definitions, the time coordinate $\dot{\tau}_{e}$ must be nonvanishing. If this condition is not satisfied we can, nevertheless, always consider that the ancillary emitter $\mathcal{S}$ generates a time number $\stackrel{\circ}{\rho}$ and broadcasts $e^{\stackrel{\circ}{\rho}} \equiv \dot{\tau}$. This can be realized from a real-time computer with $\stackrel{\circ}{\rho}$ as the generated input and $e^{\rho}$ as the broadcast output. Obviously, we can assume the same for the main emitters.

Let $g$ be an element of $G L(4, \mathbb{R})$ such that $g \cdot e_{A L}=e_{A L}^{\prime}$. And thus, $G L(4, \mathbb{R})$ acts linearly on $\mathbb{R}_{A L}^{4}$. Then, the action of $G L(4, \mathbb{R})$ on $\mathbb{R}_{L}^{4}$ and $\mathbb{R}_{P}^{3}$ is nonlinear and locally transitive and it defines homographies (i.e., conformal transformations):

$$
\begin{aligned}
e_{P}^{\prime} & =\left(\frac{A \cdot e_{P}+b}{c \cdot e_{P}+\mu}\right), \\
g & \equiv\left(\begin{array}{ll}
A & b \\
t^{t} & \mu
\end{array}\right), \\
\dot{\tau}_{e}^{\prime} & =\dot{\tau}_{e}\left(c \cdot e_{P}+\mu\right),
\end{aligned}
$$

where $\mu \in \mathbb{R},(b, c) \in\left(\mathbb{R}^{3}\right)^{2}$, and $A \in M_{3 \times 3}(\mathbb{R})$. Let us notice that $\dot{\tau}_{e}$ does not intervene in (12a). Moreover, we deduce that $P G L(4, \mathbb{R})$ acts locally transitively on $\mathbb{R}_{P}^{3}$. Therefore, we obtain the following.

Theorem 16. The $(2+1)$-dimensional spacetime manifold has a local 3-dimensional projective structure inherited from its causal structure.

Proof. Let $x, t$, and $s_{i}(i=1,2,3)$ in $\mathbb{R}_{A L}^{4}$ be such that

$$
\begin{array}{r}
x \equiv e_{\mathrm{AL}}=\left(x^{3}=\dot{\tau}_{e} \tau_{e}, x^{2}=\dot{\tau}_{e} \bar{\tau}_{e}, x^{1}=\dot{\tau}_{e} \widetilde{\tau}_{e}, x^{0}=\dot{\tau}_{e}\right), \\
t \equiv\left(t^{3}=t^{0} \tan \alpha_{e}, t^{2}=t^{0} \tan \bar{\alpha}_{e}, t^{1}=t^{0} \tan \widetilde{\alpha}_{e}, t^{0}\right), \\
\left(t^{0} \neq 0\right),
\end{array}
$$

$$
\begin{aligned}
& s_{1} \equiv\left(s_{1}^{3}=s_{1}^{0} \tau_{\delta^{*}}, s_{1}^{2}=s_{1}^{0} \tau^{\diamond}, s_{1}^{1}=s_{1}^{0} \tau^{\prime \prime}, s_{1}^{0}=\tau_{\delta^{*}} \tau^{\diamond} \tau^{\prime \prime}\right),\left(s_{1}^{0} \neq 0\right), \\
& s_{2} \equiv\left(s_{2}^{3}=s_{2}^{0} \bar{\tau}_{\delta^{*}}, s_{2}^{2}=s_{2}^{0} \bar{\tau}^{\diamond}, s_{2}^{1}=s_{2}^{0} \bar{\tau}^{\prime \prime}, s_{2}^{0}\right. \\
&\left.=\bar{\tau}_{\delta^{*}} \bar{\tau}^{\diamond} \bar{\tau}^{\prime \prime}\right), \quad\left(s_{2}^{0} \neq 0\right), \\
& s_{3} \equiv\left(s_{3}^{3}=s_{3}^{0} \widetilde{\tau}_{\delta^{\prime}}, s_{3}^{2}=s_{3}^{0} \widetilde{\tau}^{\diamond}, s_{3}^{1}=s_{3}^{0} \widetilde{\tau}^{\prime \prime}, s_{3}^{0}\right. \\
&\left.=\tilde{\tau}_{\delta^{\prime}} \widetilde{\tau}^{\diamond} \tilde{\tau}^{\prime \prime}\right), \quad\left(s_{3}^{0} \neq 0\right) .
\end{aligned}
$$

Then, the relations (1a)-(1c) can be put in the forms $(\alpha, \beta, \mu, \nu=0,1,2,3$; no summation on $i=1,2,3)$

$$
\begin{aligned}
& K_{\alpha, \beta, \mu, \nu}^{i} s_{i}^{\alpha} s_{i}^{\beta} x^{\mu} t^{\nu}=0, \\
& K_{\alpha, \beta, \mu, \nu}^{0} s_{i}^{\alpha} s_{i}^{\beta} x^{\mu} t^{\nu} \neq 0,
\end{aligned}
$$

where the coefficients of the tensors $K^{i}$ take only the values 0 or \pm 1 and the only nonvanishing coefficient of $K^{0}$ is $K_{0,0,0,0}^{0}$. Then, it is easy to show that for all $g_{x}$ and $g_{t}$ in $G L(4, \mathbb{R})$ there exists $g \in G L(4, \mathbb{R})$ such that

$$
\begin{aligned}
& K^{i}\left(g \cdot s_{i}, g \cdot s_{i}, g_{x} \cdot x, g_{t} \cdot t\right)=0, \\
& K^{0}\left(g \cdot s_{i}, g \cdot s_{i}, g_{x} \cdot x, g_{t} \cdot t\right) \neq 0 .
\end{aligned}
$$

In particular, if $g \equiv \mathrm{Id}$ and if the $s_{i}$ are fixed, then the set of localized events $x \equiv e_{A L} \in \mathbb{R}_{A L}^{4}$ is an orbit of $G L(4, \mathbb{R})$ and the set of corresponding events $e_{P}=\pi\left(e_{A L}\right)$ is an orbit of the projective group $P G L(4, \mathbb{R})$.

And then, because the relations (14) are homogeneous polynomials, we deduce that $\mathbb{R}_{A L}^{4}$ has a projective structure as expected.

Remark 17. The map $\mathbb{S}$ defined locally by $\mathfrak{M}^{-1} \times \overline{\mathfrak{M}}^{-1} \times \widetilde{\mathfrak{M}}^{-1}$ on $\mathbb{R}_{P}^{3}$ is the so-called "soldering map " $\mathbb{S}$ of Ehresmann defined on $P \mathbb{R}^{3}=\mathbb{R}_{P}^{3} \cup P \mathbb{R}^{2}$ to the spacetime manifold $\mathscr{M}$ :

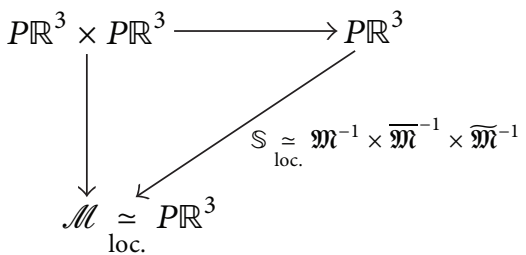

And the set of homogeneous equations $K^{i}\left(s_{i}, s_{i}, t, x\right)=0$ when the $s_{i}$ are fixed defines leaves in the trivial bundle $P \mathbb{R}^{3} \times P \mathbb{R}^{3}$. After reduction of the bundle $\mathbb{R}^{4} \times \mathbb{R}^{4} \rightarrow \mathscr{M}$ to this projective bundle, the projective Cartan connection in the sense of Ehresmann [17] is defined as the differential $d K \equiv d K^{1} \times d K^{2} \times d K^{3}$ with respect to the vertical variables $v \simeq\left(\tan \alpha_{e}, \tan \bar{\alpha}_{e}, \tan \widetilde{\alpha}_{e}\right)$ and the horizontal variables $e_{P}$; and thus, the tangent spaces of these horizontal leaves are the annihilators/contact elements of $d K$. 


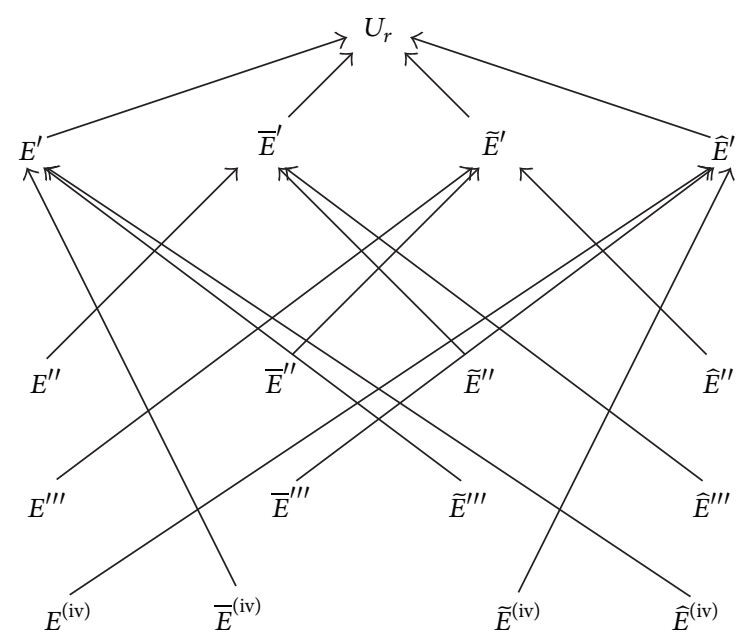

FIGURE 12: The causal structure of the RPS in a $(3+1)$-dimensional spacetime.

Remark 18. Also, as a result, the spacetime manifold can be considered as a "generalized Cartan space" which is endowed with both (1) a "projective Cartan connection" (of dimension 4) providing a local projective structure, and (2) a compatible (pseudo-)Riemannian structure viewed for instance as a horizontal section in the four-dimensional anisotropic grid.

Also, we can eventually define a Ehresmann connection providing a horizontal/vertical splitting in the tangent space of the principal bundle of projective frames of the spacetime manifold. And then, once this Ehresmann connection is given, we can define from this splitting a projective Cartan connection $^{5}$ which can be viewed as the infinitesimal changes of the projective frames with respect to themselves.

\section{RLSs in $(3+1)$-Dimensional Spacetime}

We need similarly four main emitters $\mathscr{E}, \overline{\mathscr{E}}, \widetilde{\mathscr{E}}$, and $\widehat{\mathscr{E}}$ providing a RPS and, again, one ancillary emitter $\mathcal{S}$ emitting its time coordinates and its own time stamp $\dot{\tau}$ from a clock to get a RLS.

5.1. The Causal Structure of the RPS. The protocol becomes more and more complex to implement. Indeed, sixteen time stamps are needed to provide to the users their positions in a given system of reference. These positions are obtained from the knowledge the users acquire of their own positions and those of the main emitters both in the emission grid and in the system of reference; and this is due to the ephemerides that the emitters upload to the users and the autolocating process. The causal structure of the RPS is shown in Figure 12 and Table 6.

The position in the emission grid $\mathbb{R}_{P}^{4}$ of the user at $U_{r}$ is $\left(\tau^{\prime}, \bar{\tau}^{\prime}, \widetilde{\tau}^{\prime}, \widehat{\tau}^{\prime}\right)$.

5.2. The Description of the RLS. As in the $(2+1)$-dimensional case, we need a system of light echoes associated with each privileged emitter, each linked to an event of reception on the user's worldline. Again, we denote by $\mathscr{E} \mathrm{ch}$ the system of
TABle 6: The events $E^{\prime}, \bar{E}^{\prime}, \widetilde{E}^{\prime}$, and $\widehat{E}^{\prime}$ and their broadcast time stamps received at $U_{r}$.

\begin{tabular}{lc}
\hline Event & Broadcasts time stamps \\
\hline$E^{\prime}$ & $\left(\tau^{\prime}=\tau_{E^{\prime}}, \bar{\tau}^{\text {(iv) }}, \widetilde{\tau}^{\prime \prime \prime}, \widehat{\tau}^{\text {(iv) }}\right)$ \\
$\bar{E}^{\prime}$ & $\left(\tau^{\prime \prime}, \bar{\tau}^{\prime}=\bar{\tau}_{\bar{E}^{\prime}}, \widetilde{\tau}^{\prime \prime}, \widehat{\tau}^{\prime \prime \prime}\right)$ \\
$\widetilde{E}^{\prime}$ & $\left(\tau^{\prime \prime \prime}, \bar{\tau}^{\prime \prime}, \widetilde{\tau}^{\prime}=\widetilde{\tau}_{\vec{E}}, \widehat{\tau}^{\prime \prime}\right)$ \\
$\widehat{E}^{\prime}$ & $\left(\tau^{(\mathrm{iv})}, \bar{\tau}^{\prime \prime \prime}, \widetilde{\tau}^{(\mathrm{iv})}, \widehat{\tau}^{\prime}=\widehat{\tau}_{\vec{E}}\right)$ \\
\hline
\end{tabular}

light echoes for the privileged emitter $\mathscr{E}$ with $E_{p}$ as primary event. But now, contrarily to the $(2+1)$-dimensional case, we must consider celestial spheres rather than celestial circles. And then, we have again sky mappings from the past null cones directions at the primary events to the "bright" points on the associated celestial spheres. Nevertheless, we have only homeomorphisms between hemispheres with half of their boundaries and $P \mathbb{R}^{2}$. Thus, a problem occurs a priori in this $(3+1)$-dimensional case because we have two disjoint hemispheres for each celestial sphere. And then, consecutive problems appear for the choice and the realization of these hemispheres in the localizing protocol. However, we show in the sequel this problem vanishes completely when considering the full set of echoing systems and the particular hemispheres implementation we present for the emitters. We need, first, the following definition for the determination of the first emission coordinate $\tau_{e}$.

Definition 19 (the echoing system $\mathscr{E} \mathrm{ch}$ ). The echoing system $\mathscr{E}$ ch associated with the privileged emitter $\mathscr{E}$ is based on the following features (see Figure 15):

(i) One primary event $E_{p}$ with its celestial sphere $\mathscr{C}_{E_{p}}$.

(ii) Four secondary events $\bar{E}, \widetilde{E}^{\bullet}$, and $\widehat{E}^{\bullet}$ with the ancillary event $\mathcal{S}^{\bullet}$, associated, respectively, with the canonical projective points $[\infty, 0]_{E_{p}},[0, \infty]_{E_{p}},[0,0]_{E_{p}}$, and $[1,1]_{E_{p}}$ of the projective frame $\mathfrak{F}_{E_{p}}$ defining one specific hemisphere of the celestial sphere $\mathscr{C}_{E_{p}}$ (Figure 15).

(iii) One ternary event $E^{\sharp}$ for $\vec{E}$, two ternary events $E^{\diamond}$ and $\bar{E}_{\diamond}$ for $\widetilde{E}^{\bullet}$, two ternary events $E^{\prime \prime}$ and $\bar{E}_{\prime \prime}$ for $\widehat{E}^{\bullet}$, and none for $\mathcal{S}^{\circ}$,

(iv) Two compasses on the specific hemisphere of $\mathscr{C}_{E_{p}}$ defined above with a moving origin anchored on the projective point $[1,1]_{E_{p}}$ associated with $\mathcal{S}^{\bullet}$.

(v) One event of reception $U_{r} \in \mathscr{V}$ at which all the data are collected and sent by the emitter $\mathscr{E}$.

Then, we have the following hierarchy of events in the four different echoing systems $\mathscr{E} \mathrm{ch}, \overline{\mathscr{E}} \mathrm{ch}, \widetilde{\mathscr{E}} \mathrm{ch}$, and $\widehat{\mathscr{E}} \mathrm{ch}$ :

(i) Four primary events $E_{p}, \bar{E}_{p}, \widetilde{E}_{p}$, and $\widehat{E}_{p}$, each with three secondary events and one ancillary event (Table 7).

(ii) Four horismotic relations $E_{p} \rightarrow U_{r}, \bar{E}_{p} \rightarrow \bar{U}_{r}, \widetilde{E}_{p} \rightarrow$ $\widetilde{U}_{r}$, and $\widehat{E}_{p} \rightarrow \widehat{U}_{r}$, where the chronologically ordered 
TABLE 7: The four primary events and their secondary/ancillary events with their corresponding projective points on the celestial hemispheres $\mathscr{C}_{E_{p}}, \mathscr{C}_{\bar{E}_{p}}, \mathscr{C}_{E_{p}}$, and $\mathscr{C}_{\bar{E}_{p}}$ homeomorphic to $P \mathbb{R}^{2}$.

\begin{tabular}{lccccc}
\hline Echoing system & Primary event & {$[\infty, 0]$} & {$[0, \infty]$} & {$[0,0]$} & {$[1,1]$} \\
\hline $\mathscr{E} \mathrm{ch}$ & $E_{p} \in \mathscr{W}$ & $\bar{E}^{*}$ & $\widetilde{E}^{*}$ & $\widehat{E}^{*}$ & $\mathcal{S}^{*}$ \\
$\overline{\mathscr{E}} \mathrm{ch}$ & $\bar{E}_{p} \in \mathscr{\mathscr { W }}$ & $\widetilde{E}^{\dagger}$ & $\widehat{E}^{\dagger}$ & $E^{\dagger}$ & $\mathcal{S}^{\dagger}$ \\
$\widetilde{\mathscr{E}} \mathrm{ch}$ & $\widetilde{E}_{p} \in \widetilde{\mathscr{W}}$ & $\widehat{E}^{*}$ & $E^{*}$ & $\bar{E}^{*}$ & $\mathcal{S}^{*}$ \\
$\overline{\mathscr{E}} \mathrm{ch}$ & $\widehat{E}_{p} \in \widetilde{\mathscr{W}}$ & $E^{\prime}$ & $\bar{E}^{\prime}$ & $\widetilde{E}^{\prime}$ & $\mathcal{S}^{\prime}$ \\
\hline
\end{tabular}

events of reception $U_{r}, \bar{U}_{r}, \widetilde{U}_{r}$, and $\widehat{U}_{r}$ belong to the user worldline $\mathscr{V}$.

(iii) One or two (normal and shifted) ternary events by secondary event except for the ancillary event:

$\mathscr{E} \mathrm{ch}: \bar{E}^{\bullet}: E^{\sharp}, \widetilde{E}^{\bullet}: E^{\diamond}, \bar{E}_{\diamond}, \widehat{E}^{\bullet}: E^{\prime \prime}, \bar{E}_{\prime \prime}$,

$\overline{\mathscr{E}} \mathrm{ch}: \widetilde{E}^{\dagger}: \bar{E}^{\sharp}, \widehat{E}^{\dagger}: \bar{E}^{\diamond}, \widetilde{E}_{\diamond}, E^{\dagger}: \bar{E}^{\prime \prime}, \widetilde{E}_{\prime \prime}$,

$\widetilde{\mathscr{E}} \mathrm{ch}: \widehat{E}^{*}: \widetilde{E}^{\sharp}, E^{*}: \widetilde{E}^{\diamond}, \widehat{E}_{\diamond}, \bar{E}^{*}: \widetilde{E}^{\prime \prime}, \widehat{E}_{\prime \prime}$,

$\widehat{\mathscr{E}} \mathrm{ch}: E^{\prime}: \widehat{E}^{\sharp}, \bar{E}^{\prime}: \widehat{E}^{\diamond}, E_{\diamond}, \widetilde{E}^{\prime}: \widehat{E}^{\prime \prime}, E_{\prime \prime}$.

(iv) Two events associated with the projective points $[\infty, 0]$ and $[0, \infty]$ define the equatorial circle dividing the celestial sphere $S^{2} \simeq P \mathbb{R}^{2} \# P \mathbb{R}^{2}$ in two celestial hemispheres $\mathscr{C}$ which are identified to a unique projective space $P \mathbb{R}^{2}$. In other words, the directions of propagation of the light rays detected as bright points on the hemispheres are not considered. This could be a problem a priori. Actually, this difficulty is completely canceled out from the operating principles of RLSs as we will see in the sequel.

(v) Two compasses on each celestial hemisphere $\mathscr{C}_{E_{p}}$, $\mathscr{C}_{\bar{E}_{p}}, \mathscr{C}_{\widetilde{E}_{p}}$, and $\mathscr{C}_{\widehat{E}_{p}}$ with a common moving origin for angle measurements anchored on the projective point $[1,1]$.

(vi) We recall that $\delta$ broadcasts as a particular user its own emission coordinates $\left(\tau_{S}, \bar{\tau}_{S}, \widetilde{\tau}_{S}, \bar{\tau}_{S}\right)$ obtained from the positioning system for all $S \in \mathscr{W}^{\mathcal{S}}$. It broadcasts also all along $\mathscr{W}^{\mathcal{S}}$ its own time coordinate denoted again by $\stackrel{\circ}{\tau}$.

5.3. The Causal Structure of the RLS. We represent (Figures 13 and 14 and Tables 8 and 9) only the causal structure for the echoing system $\mathscr{E} \mathrm{ch}$; the other echoing systems $\overline{\mathscr{E}} \mathrm{ch}$, $\widetilde{\mathscr{E}} \mathrm{ch}$, and $\widehat{\mathscr{E}}_{\mathrm{ch}}$ can be easily obtained from the symbolic substitutions deduced from Table 7 and (17a)-(17d).

5.4. The Projective Frame, the Time Stamps Correspondence, and the Consistency. We consider the projective frame at the primary event $E_{p}$ and the time stamps correspondence associated with the change of projective frame on $\mathscr{C}_{E_{p}}$ (Table 10 and Figure 15). Obviously, the other correspondences and changes of projective frames can be deduced in the same way for the three other primary events. Then, we obtain four corresponding pairs of time coordinates for $e$ in the four celestial hemispheres (Table 11).

Then, the change of projective frame on the celestial hemisphere gives the following relation:

$$
\begin{aligned}
& \left(\begin{array}{lll}
a & d & g \\
b & e & h \\
c & f & k
\end{array}\right)\left(\begin{array}{llllc}
1 & 0 & 0 & 1 & \rho \tan \alpha_{e} \\
0 & 1 & 0 & 1 & \rho \tan \beta_{e} \\
0 & 0 & 1 & 1 & \rho
\end{array}\right) \\
& =\left(\begin{array}{ccccc}
u \tau^{\sharp} & v \tau^{\diamond} & w \tau^{\prime \prime} & (u+v+w) \tau_{\S} \cdot & r \tau_{e}^{\cdot} \\
u \bar{\tau}^{*} & v \bar{\tau}_{\diamond} & w \bar{\tau}_{\prime \prime} & (u+v+w) \bar{\tau}_{\delta} \cdot & r \bar{\tau}_{e}^{\cdot} \\
u & v & w & (u+v+w) & r
\end{array}\right),
\end{aligned}
$$

where $\rho u v w r(u+v+w) \neq 0$, and where the determinant of the square matrix on the l.h.s. of this equality must be nonvanishing. Then, we deduce that

$$
\begin{gathered}
\left(\begin{array}{ccc}
u \tau^{\sharp} & v \tau^{\diamond} & w \tau^{\prime \prime} \\
u \bar{\tau}^{\bullet} & v \bar{\tau}_{\diamond} & w \bar{\tau}_{\prime \prime} \\
u & v & w
\end{array}\right)\left(\begin{array}{cc}
1 & \rho \tan \alpha_{e} \\
1 & \rho \tan \beta_{e} \\
1 & \rho
\end{array}\right) \\
=r(u+v+w)\left(\begin{array}{cc}
\tau_{\delta^{*}} & \tau_{e}^{\cdot} \\
\bar{\tau}_{\delta^{*}} & \bar{\tau}_{e}^{\cdot} \\
1 & 1
\end{array}\right),
\end{gathered}
$$

and we can take in addition $u+v+w=r=1$.

Obviously, we obtain from the three other echoing systems three other similar systems of equations for the other six unknown time coordinates given in Table 11 for $e$.

Now, besides, we have necessarily the relations:

$$
\begin{aligned}
& \tau_{e}^{\cdot}=\tau_{e}^{\prime}, \\
& \bar{\tau}_{e}^{*}=\bar{\tau}_{e}^{\dagger}, \\
& \tilde{\tau}_{e}^{*}=\widetilde{\tau}_{e}^{\dagger}, \\
& \widehat{\tau}_{e}^{*}=\widehat{\tau}_{e}^{\prime} .
\end{aligned}
$$

Indeed, if one of these four precedent equalities is not satisfied, then it means that if the event $e$, the worldlines of the main emitters, and the ancillary one are fixed, then, at least one time stamp among the eight can vary. And then, one of 


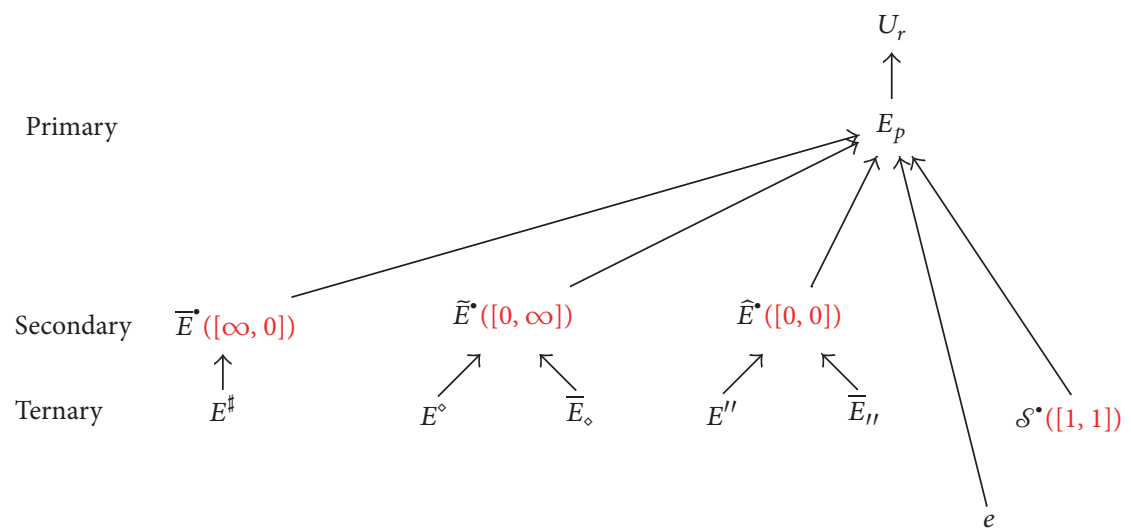

$E^{\sharp}, E^{\diamond}$ and $E^{\prime \prime}$ chronologically ordered, and $\bar{E}_{\diamond}$ and $\bar{E}_{/ \prime}$ chronologically ordered.

FIGURE 13: The echoing system $\mathscr{E} \mathrm{ch}$.

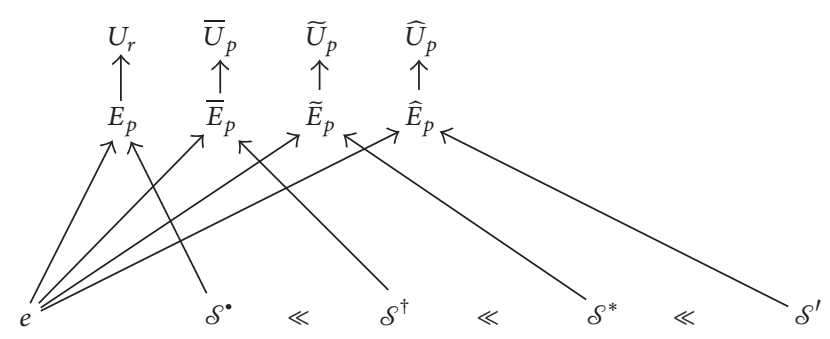

Figure 14: The causal structure for the four echoing systems $\mathscr{E} \mathrm{ch}$, $\overline{\mathscr{E}} \mathrm{ch}, \widetilde{\mathscr{E}} \mathrm{ch}$, and $\widehat{\mathscr{E}} \mathrm{ch}$ with the event $e$. The chronological order between $\mathcal{S}^{\bullet}, \mathcal{S}^{\dagger}, \mathcal{S}^{*}$, and $\mathcal{S}^{\prime}$ belonging to $\mathscr{W}^{\mathcal{S}}$ can be different.

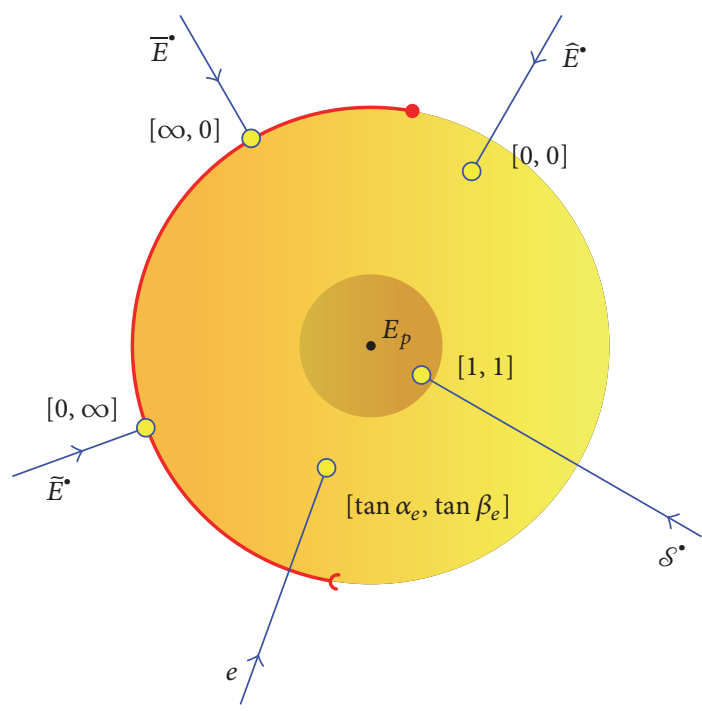

Figure 15: The projective disk on the celestial hemisphere $\mathscr{C}_{E_{p}}$ centered at $E_{p}$ and the four canonical projective points and the corresponding projective point for $e$.

the eight angles on the four celestial hemispheres necessarily can vary as well. But then, it would mean that the position of the event $e$ seen on the celestial hemispheres of the four main emitters can vary arbitrarily whenever $e$ is fixed. In other
TABLE 8: The secondary/ancillary events and their broadcast time stamps in the $\mathscr{E} \mathrm{ch}$ system.

\begin{tabular}{lcc}
\hline Event & Broadcasts time stamps & Received at \\
\hline$\vec{E}$ & $\left(\tau^{\sharp}, \bar{\tau}^{\bullet}=\bar{\tau}_{\vec{E}^{*}}\right)$ & $E_{p}$ \\
$\widetilde{E}^{\cdot}$ & $\left(\tau^{\diamond}, \bar{\tau}_{\diamond}\right)$ & $E_{p}$ \\
$\widehat{E}^{\cdot}$ & $\left(\tau^{\prime \prime}, \bar{\tau}_{\prime \prime}\right)$ & $E_{p}$ \\
$\mathcal{S}^{\bullet}$ & $\left(\tau_{\delta^{*}}, \bar{\tau}_{\delta^{*}}\right)$ & $E_{p}$ \\
\hline
\end{tabular}

TABLE 9: The ternary events and their broadcast time stamps in the $\mathscr{E}$ ch system.

\begin{tabular}{lcc}
\hline Event & Broadcasts time stamps & Received at \\
\hline$E^{\sharp}$ & $\tau^{\sharp}$ & $\vec{E}$ \\
$E^{\diamond}$ & $\tau^{\diamond}$ & $\widetilde{E}$ \\
$\bar{E}_{\diamond}$ & $\bar{\tau}_{\diamond}$ & $\widetilde{E}$ \\
$E^{\prime \prime}$ & $\tau^{\prime \prime}$ & $\widehat{E}$ \\
$\bar{E}_{\prime \prime}$ & $\bar{\tau}_{\prime \prime}$ & $\widehat{E}$ \\
\hline
\end{tabular}

words, $e$ might have more than one corresponding "bright" point on each celestial hemisphere; and, in particular, because we have continuous functions, then it might correspond to $e$, in particular, a connected "bright" line on one of the four celestial hemispheres. This would involve necessarily the existence of more than one and only one horismotic relation " $\rightarrow$." This situation can be encountered in the case of the existence of conjugate points for light-like geodesics for instance in Riemannian manifolds. Then, considering only one horismos, the relations (20) must be satisfied.

Then, we obtain the following.

Lemma 20. Let $\mathscr{T}^{4} \equiv\left(P \mathbb{R}^{1}\right)^{4}$ be the 4-torus. Then, the RLS provides a map

$$
\begin{array}{r}
\mathfrak{M}_{4}:\left(\tan \alpha_{e}, \tan \bar{\alpha}_{e}, \tan \tilde{\alpha}_{e}, \tan \widehat{\alpha}_{e}\right) \in \mathscr{T}^{4} \longrightarrow \\
\left(\tau_{e}, \bar{\tau}_{e}, \widetilde{\tau}_{e}, \widehat{\tau}_{e}\right) \in \mathscr{T}^{4}
\end{array}
$$

which is an automorphism. 
TABLE 10: The change of projective frame and the corresponding events.

\begin{tabular}{lcc}
\hline Event & $\mathfrak{\mho}_{E_{p}}$ & $\mathfrak{F}_{E_{p}}^{\tau}$ \\
\hline$e$ & {$\left[\tan \alpha_{e}, \tan \beta_{e}\right]$} & {$\left[\tau_{e}^{\cdot}, \bar{\tau}_{e}^{*}\right]$} \\
$\vec{E}$ & {$[\infty, 0]$} & {$\left[\tau^{\sharp}, \bar{\tau}^{\bullet}=\bar{\tau}_{\bar{E}^{\cdot}}\right]$} \\
$\widetilde{E}^{\cdot}$ & {$[0, \infty]$} & {$\left[\tau^{\diamond}, \bar{\tau}_{\diamond}\right]$} \\
$\widehat{E}^{\cdot}$ & {$[0,0]$} & {$\left[\tau^{\prime \prime}, \bar{\tau}_{\prime \prime}\right]$} \\
$\mathcal{S}^{\cdot}$ & {$[1,1]$} & {$\left[\tau_{\mathcal{S}^{*}}, \bar{\tau}_{\mathcal{S}^{*}}\right]$} \\
\hline
\end{tabular}

TABLE 11: The pairs of time coordinates for $e$ deduced in the four celestial hemispheres.

\begin{tabular}{lc}
\hline Celestial hemisphere & Time coordinates for $e$ \\
\hline $\mathscr{C}_{E_{p}}$ & $\left(\tau_{e}^{*}, \vec{\tau}_{e}\right)$ \\
$\mathscr{C}_{\bar{E}_{p}}$ & $\left(\bar{\tau}_{e}^{\dagger}, \widetilde{\tau}_{e}^{\dagger}\right)$ \\
$\mathscr{C}_{\widetilde{E}_{p}}$ & $\left(\widetilde{\tau}_{e}^{*}, \widetilde{\tau}_{e}^{*}\right)$ \\
$\mathscr{C}_{\bar{E}_{p}}$ & $\left(\widehat{\tau}_{e}^{\prime}, \tau_{e}^{\prime}\right)$ \\
\hline
\end{tabular}

Proof. This lemma can be easily proved simply by solving systems of equations like (19) but we indicate interesting intermediate homogeneous equations in the computations. From the relations (20) and the equations at each primary event such as (19) at $E_{p}$, we deduce that there are four linear relations between " $\tan \alpha$ " and " $\tan \beta$. ." And then, it can be shown that we obtain four Möbius relations linking the four $\tan \alpha$ 's to the four time coordinates $\tau_{e}, \bar{\tau}_{e}, \widetilde{\tau}_{e}$, and $\widehat{\tau}_{e}$ of $e$ generalizing the situation encountered in the precedent $(2+$ 1)-dimensional case.

More precisely, considering the primary event $E_{p}$, we obtain (19). At the other primary event $\widetilde{E}_{p}$, we obtain the similar following relations $(\nu \neq 0)$ :

$$
\begin{gathered}
\left(\begin{array}{ccc}
p \widetilde{\tau}^{\sharp} & q \widetilde{\tau}^{\diamond} & m \widetilde{\tau}^{\prime \prime} \\
p \widetilde{\tau}^{*} & q \widehat{\tau}_{\diamond} & m \widehat{\tau}_{\prime \prime} \\
p & q & m
\end{array}\right)\left(\begin{array}{cc}
1 & v \tan \widetilde{\alpha}_{e} \\
1 & v \tan \widetilde{\beta}_{e} \\
1 & v
\end{array}\right) \\
=n(p+q+m)\left(\begin{array}{cc}
\tilde{\tau}_{\delta^{*}} & \widetilde{\tau}_{e}^{*} \\
\hat{\tau}_{\delta^{*}} & \widehat{\tau}_{e}^{*} \\
1 & 1
\end{array}\right),
\end{gathered}
$$

where again we can impose the relations $n=p+q+m=1$. Then, from now and throughout, we set

$$
\begin{aligned}
& \tau_{e} \equiv \tau_{e}^{\cdot}=\tau_{e}^{\prime}, \\
& \bar{\tau}_{e} \equiv \vec{\tau}_{e}^{\cdot}=\bar{\tau}_{e}^{\dagger}, \\
& \widetilde{\tau}_{e} \equiv \widetilde{\tau}_{e}^{*}=\widetilde{\tau}_{e}^{\dagger}, \\
& \widehat{\tau}_{e} \equiv \widehat{\tau}_{e}^{*}=\widehat{\tau}_{e}^{\prime} .
\end{aligned}
$$

And then, it can be shown that the relations (19) and (22), those depending explicitly on the time stamps, can be put in the following forms $(p, q=1, \ldots, 4$ and $\mu, \nu=0,1, \ldots, 4)$ :

$$
\begin{aligned}
& K_{p q \mu \nu} s^{p} S^{q} x^{\mu} t^{\nu}=0, \\
& \bar{K}_{p q \mu \nu} \bar{s}^{p} S^{q} x^{\mu} t^{\nu}=0, \\
& \widetilde{K}_{p q \mu \nu} \widetilde{s}^{p} \widetilde{S}^{q} x^{\mu} t^{\nu}=0, \\
& \widehat{K}_{p q \mu \nu} \widehat{s}^{p} \widetilde{S}^{q} x^{\mu} t^{\nu}=0,
\end{aligned}
$$

where

$$
\begin{aligned}
& x \equiv\left(\dot{\tau}_{e} \tau_{e}, \dot{\tau}_{e} \bar{\tau}_{e}, \dot{\tau}_{e} \widetilde{\tau}_{e}, \stackrel{\tau}{\tau}_{e} \widehat{\tau}_{e}, \stackrel{\circ}{\tau}_{e}\right), \\
& t \equiv(\mu \tan \alpha, \mu \tan \beta, \mu \tan \widetilde{\alpha}, \mu \tan \widetilde{\beta}, \mu) \text {, } \\
& s \equiv\left(\tau^{\sharp}, \tau^{\diamond}, \tau^{\prime \prime}, s^{0}\right), \\
& \bar{s} \equiv\left(\bar{\tau}^{\bullet}, \bar{\tau}_{\diamond}, \bar{\tau}_{\prime \prime}, \bar{s}^{0}=s^{0}\right), \\
& \widetilde{s} \equiv\left(\widetilde{\tau}^{\sharp}, \widetilde{\tau}^{\diamond}, \widetilde{\tau}^{\prime \prime}, \widetilde{s}^{0}\right), \\
& \widehat{s} \equiv\left(\widehat{\tau}^{*}, \widehat{\tau}_{\diamond}, \widehat{\tau}_{11}, \widehat{s}^{0}=\widetilde{s}^{0}\right), \\
& S \equiv\left(\left|\begin{array}{ccc}
\tau_{\delta^{*}} & \tau^{\diamond} & \tau^{\prime \prime} \\
\bar{\tau}_{\mathcal{S}^{*}} & \bar{\tau}_{\diamond} & \bar{\tau}_{\prime \prime} \\
1 & 1 & 1
\end{array}\right|,\left|\begin{array}{ccc}
\tau^{\sharp} & \tau_{\mathcal{S}^{\bullet}} & \tau^{\prime \prime} \\
\bar{\tau}^{\bullet} & \bar{\tau}_{\mathcal{S}^{\bullet}} & \bar{\tau}_{\prime^{\prime \prime}} \\
1 & 1 & 1
\end{array}\right|,\left|\begin{array}{ccc}
\tau^{\sharp} & \tau^{\diamond} & \tau_{\delta^{\bullet}} \\
\bar{\tau}^{\bullet} & \bar{\tau}_{\diamond} & \bar{\tau}_{\mathcal{S}^{\bullet}} \\
1 & 1 & 1
\end{array}\right|,\right. \\
& \left.\left|\begin{array}{ccc}
\tau^{\sharp} & \tau^{\diamond} & \tau^{\prime \prime} \\
\bar{\tau}^{\bullet} & \bar{\tau}_{\diamond} & \bar{\tau}^{\prime \prime} \\
1 & 1 & 1
\end{array}\right|\right) \\
& \widetilde{S} \equiv\left(\left|\begin{array}{ccc}
\tilde{\tau}_{\delta^{*}} & \widetilde{\tau}^{\diamond} & \tilde{\tau}^{\prime \prime} \\
\hat{\tau}_{\delta^{*}} & \widehat{\tau}_{\diamond} & \widehat{\tau}_{\prime \prime} \\
1 & 1 & 1
\end{array}\right|,\left|\begin{array}{ccc}
\tilde{\tau}^{\sharp} & \tilde{\tau}_{\delta^{*}} & \tilde{\tau}^{\prime \prime} \\
\widehat{\tau}^{*} & \widehat{\tau}_{\delta^{*}} & \widehat{\tau}_{\prime^{\prime \prime}} \\
1 & 1 & 1
\end{array}\right|,\left|\begin{array}{ccc}
\tilde{\tau}^{\sharp} & \widetilde{\tau}^{\diamond} & \tilde{\tau}_{\delta^{*}} \\
\widehat{\tau}^{*} & \widehat{\tau}_{\diamond} & \widehat{\tau}_{\delta^{*}} \\
1 & 1 & 1
\end{array}\right|,\right. \\
& \left.\left|\begin{array}{ccc}
\tilde{\tau}^{\sharp} & \widetilde{\tau}^{\diamond} & \tilde{\tau}^{\prime \prime} \\
\widehat{\tau}^{*} & \widehat{\tau}_{\diamond} & \widehat{\tau}_{\prime \prime} \\
1 & 1 & 1
\end{array}\right|\right)
\end{aligned}
$$

where $s^{0}$ and $\widetilde{s}^{0}$ are nonvanishing arbitrary coefficients and $\mu \neq 0$. Hence, we have four homogeneous algebraic equations linking the vectors $t$ and $x$. Obviously, we have also four other similar homogeneous equations for $x$ and another $t^{\prime} \simeq$ $(\tan \bar{\alpha}, \tan \bar{\beta}, \tan \hat{\alpha}, \tan \widehat{\beta})$ deduced from the echoing systems at the other two primary events $\bar{E}_{p}$ and $\widehat{E}_{p}$ :

$$
\begin{aligned}
& K_{p q \mu \nu} s^{\prime p} \bar{S}^{q} x^{\mu} t^{\prime v}=0, \\
& \bar{K}_{p q \mu \nu} \bar{s}^{\prime p} \bar{S}^{q} x^{\mu} t^{\prime \nu}=0, \\
& \widetilde{K}_{p q \mu \nu} \widetilde{s}^{\prime} p \widehat{S}^{q} x^{\mu} t^{\prime \nu}=0, \\
& \widehat{K}_{p q \mu \nu} \widehat{s}^{\prime p} \widehat{S}^{q} x^{\mu} t^{\prime v}=0,
\end{aligned}
$$


where $s^{\prime 0}=\bar{s}^{\prime 0}$ and ${\widetilde{s}^{\prime 0}}^{\prime 0} \widehat{s}^{\prime 0}$ are nonvanishing arbitrary coefficients. And then, because $x$ is determined completely from (24a)-(24b), (27a)-(27b) are linearly depending on (24a)(24b) which involves that we have linear relations between the two sets of "angles" $t \simeq(\tan \alpha, \tan \beta, \tan \widetilde{\alpha}, \tan \widetilde{\beta})$ and $t^{\prime} \simeq$ $(\tan \bar{\alpha}, \tan \bar{\beta}, \tan \widehat{\alpha}, \tan \widehat{\beta})$. Hence, taking linear combinations of the systems of equations (27a)-(27b) and (24a)-(24b) and taking into account also the remaining equations in (19) and (22) not depending on the time stamps we can deduce a system of four homogeneous equations linking $y \equiv$ $(\tan \alpha, \tan \bar{\alpha}, \tan \widetilde{\alpha}, \tan \widehat{\alpha}, 1)$ and $z \equiv\left(\tau_{e}, \bar{\tau}_{e}, \widetilde{\tau}_{e}, \widehat{\tau}_{e}, 1\right):$

$$
H_{\mu, \nu}^{i} y^{\mu} z^{\nu}=0, \quad(i=1,2,3,4)
$$

where $s^{0}, \widetilde{s}^{0}, s^{\prime 0}$, and $\widetilde{s}^{\prime 0}$ do not intervene anymore. And (28) determines univocally $x$ up to the time coordinate $\dot{\tau}_{e}$, that is, $\left(\tau_{e}, \bar{\tau}_{e}, \widetilde{\tau}_{e}, \widehat{\tau}_{e}\right)$. Moreover, (28) is another expression for Möbius transformations between each given angle and a linear combination of $\left(\tau_{e}, \bar{\tau}_{e}, \widetilde{\tau}_{e}, \widehat{\tau}_{e}\right)$, hence the result for an automorphism on the 4 -torus.

Remark 21. We can notice from Lemma 20 that we obtain the time coordinates $\left(\tau_{e}, \bar{\tau}_{e}, \widetilde{\tau}_{e}, \widehat{\tau}_{e}\right)$ for $e$ from only two echoing systems, for example, at $E_{p}$ and $\widetilde{E}_{p}$ with the four "angles" ( $\tan \alpha, \tan \beta, \tan \widetilde{\alpha}, \tan \widetilde{\beta})$, or from the four echoing systems at $E_{p}, \bar{E}_{p}, \widetilde{E}_{p}$, and $\widehat{E}_{p}$ with the four "angles" $(\tan \alpha, \tan \bar{\alpha}, \tan \widetilde{\alpha}, \tan \widehat{\alpha})$.

Theorem 22. The localization and the positioning protocols or systems in a $(3+1)$-dimensional spacetime are consistent.

Proof. The proof is obvious because (1) the RLS in the $(3+$ 1)-dimensional case has a causal structure which can be decomposed in four causal substructures each equivalent to the one given for the RLS in the $(2+1)$-dimensional case and (2) we need only the "angles" $\alpha$ to localize $e$ in each of these subsystems of localization.

\subsection{The Local Projective Structure}

Definition 23. We call

(i) Emission grid the Euclidean space $\mathbb{R}_{P}^{4} \equiv \mathbb{R}^{4}$ of the positioned events $e_{P}=\left(\tau_{e}, \bar{\tau}_{e}, \widetilde{\tau}_{e}, \widehat{\tau}_{e}\right)$;

(ii) Localization (or pentametric) grid the Euclidean space $\mathbb{R}_{L}^{5} \equiv \mathbb{R}^{4} \times \mathbb{R}^{*}$ of the localized events $e_{L}=$ $\left(\tau_{e}, \bar{\tau}_{e}, \widetilde{\tau}_{e}, \widehat{\tau}_{e}, \dot{\tau}_{e}\right)$ where $\dot{\tau}_{e}$ is provided by the ancillary emitter $\delta$ by identification from the horismotic relation $S^{L} \rightarrow e_{P}\left(S^{L} \in \mathscr{W}^{\mathcal{S}}\right)$ or the "message function" [11] $f_{\mathcal{S}}^{-}: \mathbb{R}_{P}^{4} \rightarrow \mathscr{W}^{\mathcal{S}}$; that is, the time stamp $\stackrel{\circ}{\tau}^{L}$ broadcast by $\mathcal{S}$ at $S^{L}$ is such that $\stackrel{\circ}{\tau}^{L} \equiv \stackrel{\circ}{\tau}_{e}$;

(iii) Anisotropic localization (or pentametric) grid the Euclidean space $\mathbb{R}_{A L}^{5} \equiv \mathbb{R}^{4} \times \mathbb{R}^{*}$ of events $e_{A L}=$ $\left(\stackrel{\circ}{\tau}_{e} \tau_{e}, \dot{\tau}_{e} \bar{\tau}_{e}, \dot{\tau}_{e} \widetilde{\tau}_{e}, \dot{\tau}_{e} \widehat{\tau}_{e}, \dot{\tau}_{e}\right)$.
Definition 24. We denote by $I: \mathbb{R}_{L}^{5} \rightarrow \mathbb{R}_{A L}^{5}$ the bijective map such that $I\left(e_{L}\right)=e_{A L}$. And we denote by $\pi: \mathbb{R}_{A L}^{5} \rightarrow \mathbb{R}_{P}^{4}$ the submersion such that $\pi\left(e_{A L}\right)=e_{P}$.

Let $g$ be an element of $G L(5, \mathbb{R})$ such that $g \cdot e_{A L}=$ $e_{A L}^{\prime}$. And thus, $G L(5, \mathbb{R})$ acts linearly on $\mathbb{R}_{A L}^{5}$. Then, the action of $G L(5, \mathbb{R})$ on $\mathbb{R}_{L}^{5}$ and $\mathbb{R}_{P}^{4}$ defines homographies (i.e., conformal transformations):

$$
\begin{aligned}
e_{P}^{\prime} & =\left(\frac{A \cdot e_{P}+b}{c \cdot e_{P}+\mu}\right), \\
g & \equiv\left(\begin{array}{ll}
A & b \\
{ }^{t} c & \mu
\end{array}\right), \\
\stackrel{\circ}{\tau}_{e}^{\prime} & =\stackrel{\circ}{\tau}_{e}\left(c \cdot e_{P}+\mu\right),
\end{aligned}
$$

where $\mu \in \mathbb{R},(b, c) \in\left(\mathbb{R}^{4}\right)^{2}$, and $A \in M_{4 \times 4}(\mathbb{R})$. Therefore, we obtain the following.

Theorem 25. The $(3+1)$-dimensional spacetime manifold has a local 4-dimensional projective structure inherited from its causal structure.

Proof. The proof is similar with the proof of Theorem 16 but with the systems of homogeneous equations (24a)-(24b) or (27a)-(27b) or (28) instead of the system (14).

Remark 26. The map $\mathbb{S}$ defined locally by $\mathfrak{M}_{4}$ on $\mathbb{R}_{P}^{4}$ is the so-called "soldering map" $\mathbb{S}$ of Ehresmann defined on $P \mathbb{R}^{4}=$ $\mathbb{R}_{P}^{4} \cup P \mathbb{R}^{3}$ to the spacetime manifold $\mathscr{M}$ :

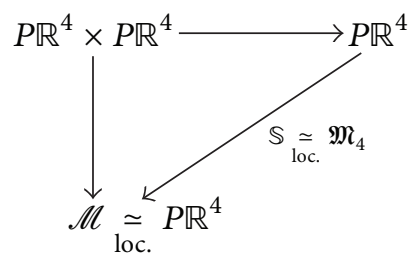

And the set of homogeneous equations (24a)-(24b) or (27a)-(27b) or (28) defines leaves in the trivial bundle $P \mathbb{R}^{4} \times$ $P \mathbb{R}^{4}$. After reduction of the bundle $\mathbb{R}^{5} \times \mathbb{R}^{5} \rightarrow \mathscr{M}$ to this projective bundle, the projective Cartan connection in the sense of Ehresmann [17] is defined as the differential $d H \equiv$ $d H^{1} \times d H^{2} \times d H^{3} \times d H^{4}$ with respect to the vertical variables $v \simeq(\tan \alpha, \tan \bar{\alpha}, \tan \tilde{\alpha}, \tan \widehat{\alpha})$ and the horizontal variables $e_{P} ;$ and thus, the tangent spaces of these horizontal leaves are the annihilators/contact elements of $d H$.

Remark 27. As in the $(2+1)$-dimensional case (see Remark 18), the spacetime manifold can be considered as a "generalized Cartan space" which is endowed with both (1) a "projective Cartan connection" (of dimension 5) providing a local projective structure and (2) a compatible (pseudo-) Riemannian structure viewed for instance as a horizontal section in the five-dimensional anisotropic grid. 


\section{Conclusion}

The previous results are obtained on manifolds of dimension less than or equal to four and satisfying only causal axiomatics. This involves only the following assumptions:

(i) A finite speed of light from the existence of the horismotic relation " $\rightarrow$,"

(ii) Isotropy (compasses) because the conformal invariance is a common consequence of all the causal axiomatics (Malament's Theorem [13], Woodhouse's axiomatics [11], King-Hawking-McCarty's axiomatics [12], e.g.; see also [10]).

(iii) Homogeneity is void of meaning in causal axiomatics.

And then, we deduced that

(i) the spacetime manifold has a local projective structure in addition to the global (pseudo-)Riemannian structure.

(ii) The spacetime manifold is a "generalized Cartan space" with a "projective Cartan connection" (see Remarks 18 and 27). A forthcoming publication is planned to clarify these aspects.

(iii) The space and time coordinates are locally transformed by homographies. Indeed, the time stamps $\left(\tau^{\alpha}\right)=\left(\tau_{e}, \bar{\tau}_{e}, \widetilde{\tau}_{e}, \widehat{\tau}_{e}\right)(\alpha=1, \ldots, 4)$ are the "emission coordinates." Then, they define a null coframe $\left(d \tau^{\alpha}\right)$ also called "null GPS coordinates" which are linearly related to timelike (GPS) coordinates [5] such as the usual space and time coordinates $\left(u^{\alpha}\right) \equiv(x, y, z, t)$. Therefore, we obtain transformations similar to the transformations (29a) for the space and time coordinates:

$$
u^{\alpha}=\left(\frac{U_{\beta}^{\alpha} u^{\prime \beta}+v^{\alpha}}{w_{\mu} u^{\prime \mu}+\rho}\right), \quad \alpha, \beta, \mu=1, \ldots, 4 .
$$

Besides, applying by inquisitiveness these projective aspects in astrophysics, we consider a modification of the Newton's law of gravitation by a homography:

$$
\vec{F}\left(\vec{r}_{0}\right) \equiv-G \frac{m_{0} m}{r_{0}^{2}}\left(\alpha+\beta t+\mu r_{0}\right)^{2} \widehat{r}_{0}, \quad \widehat{r}_{0}=\frac{\vec{r}_{0}}{r_{0}},
$$

where we consider that $\vec{F}$, the time $t$, and the radial distance $r_{0}$ between the punctual masses $m_{0}$ and $m$ are evaluated with respect to a frame attached to $m$, and $\alpha, \beta$, and $\mu$ are constants.

This modification differs from those investigated in MOND theories which satisfy the so-called Milgrom's law [18]. Also, contrarily to MOND theories, the present modification of Newton's law preserves the action/reaction principle. This modification is based on the notion of projective tensor differing from the usual notion of Euclidean tensor.

We can quote É. Cartan on this notion of Euclidean tensor [19, Section 23, p. 22]. The latter can be considered as a set of numbers $\left(u^{1}, u^{2}, \ldots, u^{r}\right)$ brought into coincidence with another set of numbers $\left(u^{\prime 1}, u^{\prime 2}, \ldots, u^{\prime r}\right)$ by a linear transformation $S_{o}$ corresponding to a rotation $R_{o}$ in a given "underlying" Euclidean space $\mathbb{R}^{k}$. Then, the linear transformation $S_{o}$ corresponds to another transformation $R_{o}$ preserving the origin $o$ of $\mathbb{R}^{k}$. Thus, we obtain tensors at this origin.

Now, a projective tensor can be considered as a set of numbers $\left(v^{1}, v^{2}, \ldots, v^{r}\right)$ brought into coincidence with another set of numbers $\left(v^{\prime 1}, v^{\prime 2}, \ldots, v^{\prime r}\right)$ by a linear transformation $S_{o}$ corresponding to an homography $H_{o}$ preserving the origin $o\left(H_{o}\right.$ is then a central collineation of center $\left.o\right)$ of a given "underlying" Euclidean space $\mathbb{R}^{k}$. In other words, the equivariance is defined for Euclidean tensors with respect to linear groups of transformations whereas the equivariance for projective tensors is defined with respect to the group of central collineations which is a subgroup of the projective group.

Also, if we have tensor fields, that is, tensors at different origins $p$ elements of a manifold (as space of parameters), then, there correspond fields (or families) of transformations $S_{p}$ on this manifold associated with fields of rotations $R_{o, p}$ (Euclidean tensor fields) or fields of central collineations $H_{o, p}$ (projective tensor fields) associated with the origin $o$ of the underlying Euclidean space $\mathbb{R}^{k}$. Then, the tensor fields are equivariant if and only if the equivariance is satisfied at any point $p$. We recognize in this description the structure of a tensor bundle of rank $k$ of which the transition morphisms (functions) are the rotation or the collineation fields, the transformation fields $S_{p}$ are defined from the local trivializations of the bundle, and the origin $o$ is an element of the fiber. Moreover, the equivariance of tensor fields is obviously the so-called left-invariance with respect to right actions of structural groups.

Then, we consider, first, the (non-modified) force of gravitation $\vec{F}$ as a Euclidean vector field with a spherical symmetry with respect to the point $p_{0}$ where the mass $m_{0}$ is located. The mass $m$ is at the point $p$ and, as indicated previously, $\vec{F}$ and $\vec{r}_{0}$ are evaluated with respect to a Euclidean frame attached to $p$. Then, clearly, rotating this frame does not change $r_{0}$ and it rotates in the same way the vector $\widehat{r}_{0}$.

Second, if $\vec{F}$ is modified to be a projective vector field with a spherical symmetry with respect to $p_{0}$, we must proceed as follows. In this projective framework, the central collineation fields $H_{o, p}$ are defined such that at each $p$ they are particular changes of projective frames $\mathfrak{F}_{p}$. More precisely, we recall that the projective frames of a projective space of dimension four are defined by six vectors in a vector space of dimension five of which five are linearly independent. Then, projective transformations, that is, homographies, are the linear, injective transformations in this five-dimensional vector space which is also called the space of homogeneous coordinates. Then, central collineations are those projective transformations preserving, up to a multiplicative factor, a particular, given five-dimensional vector, that is, the origin $o$ of the five-dimensional fiber. In general, this vector is chosen among the vectors of a given projective frame. Moreover, $p$ can be kept invariant with respect to central collineations which constitute a subgroup of the group of projective 
transformations. Indeed, these central collineations can also be viewed as local changes of inhomogeneous coordinates centered at $p$, that is, $p$ is the origin of the local system of coordinates. Hence, if $(x, y, z, t)$ are space and time coordinates centered at $p$ such that $p \equiv(0,0,0,0)=\left(x_{p}, y_{p}, z_{p}, t_{p}\right)$, then the changes of coordinates we must consider are given by the homographies (31) where $v^{\alpha}=0(\alpha=1, \ldots, 4)$; that is, we have central collineations $H_{p, o}:\left(u^{\prime \alpha}\right) \equiv\left(x^{\prime}, y^{\prime}, z^{\prime}, t^{\prime}\right) \rightarrow$ $\left(u^{\alpha}\right) \equiv(x, y, z, t)$ such that

$$
u^{\alpha}=\frac{U_{\beta}^{\alpha} u^{\prime \beta}}{\left(q+h t^{\prime}+\vec{k} \cdot \overrightarrow{r^{\prime}}\right)},
$$

where $\overrightarrow{r^{\prime}} \equiv\left(u^{\prime i}\right) \equiv\left(x^{\prime}, y^{\prime}, z^{\prime}\right)(i, j, \ldots=1,2,3),\left(U_{\beta}^{\alpha}\right)$ is a matrix field, $q$ and $h$ are scalar fields, and $\vec{k}$ is a vector field all of them depending on $p$.

In particular, if the time and space splitting of the Newtonian physics is maintained in this change of coordinates, then we must have $U_{4}^{i}=U_{i}^{4}=0$. And then, we deduce in particular that

$$
\begin{aligned}
& r=\lambda \frac{r^{\prime}}{\left(q+h t^{\prime}+\vec{k} \cdot \overrightarrow{r^{\prime}}\right)}, \\
& t=\mu \frac{t^{\prime}}{\left(q+h t^{\prime}+\vec{k} \cdot \overrightarrow{r^{\prime}}\right)},
\end{aligned}
$$

where $\lambda=\operatorname{det}\left(U_{j}^{i}\right)$ and $\mu=U_{4}^{4}$. Also, from (33), considering that (1) $\vec{F}$ is a projective five-vector with two vanishing components, that is, $\vec{F} \equiv\left(F^{1}, F^{2}, F^{3}, 0,0\right)$, and (2) the central collineations are represented (or, are originated from) by the linear transformations $U \equiv\left(U_{b}^{a}\right)(a, b=1, \ldots, 5)$ such that $U_{5}^{a}=0, U_{i}^{5}=k^{i}(i=1, \ldots, 3), U_{4}^{5}=h$, and $U_{5}^{5}=q$, then we obtain in particular $F^{i} \equiv \sum_{j=1}^{3} U_{j}^{i} F^{\prime j}$.

Then, it is easy to see that (32) becomes equivariant with respect to these changes of coordinates if and only if we set the necessary but, nevertheless, sufficient condition that the vector field $\vec{k}$ satisfies the relation $\vec{k} \equiv \sigma r_{0}^{\prime} / r_{0}^{\prime}$, where $\sigma$ is a scalar field depending on $p$ and $\overrightarrow{r_{0}^{\prime}}$ is the vector from $p$ to $p_{0}$ in the new system of coordinates. Indeed, with this condition, we obtain the new Newtonian force:

$$
\overrightarrow{F^{\prime}}\left(\overrightarrow{r_{0}^{\prime}}\right) \equiv-G \frac{m_{0} m}{r_{0}^{\prime 2}}\left(\alpha^{\prime}+\beta^{\prime} t^{\prime}+\mu^{\prime} r_{0}^{\prime}\right)^{2} \widehat{r}_{0}^{\prime}, \quad \widehat{r}_{0}^{\prime}=\frac{\overrightarrow{r_{0}^{\prime}}}{r_{0}^{\prime}} .
$$

More precisely, the equivariance is obtained as soon as $\overrightarrow{r^{\prime}}$ is equal to $\overrightarrow{r_{0}^{\prime}}$, that is, when we move along the line joining $p$ and $p_{0}$, and then, $\vec{F}$ is an equivariant, projective vector field along this line onto which only the Newton's law is experimentally evaluated. Also, we obtain the field of transformations $S_{p}$ as expected and a justification of the modification (32) of the Newton's law of gravitation.

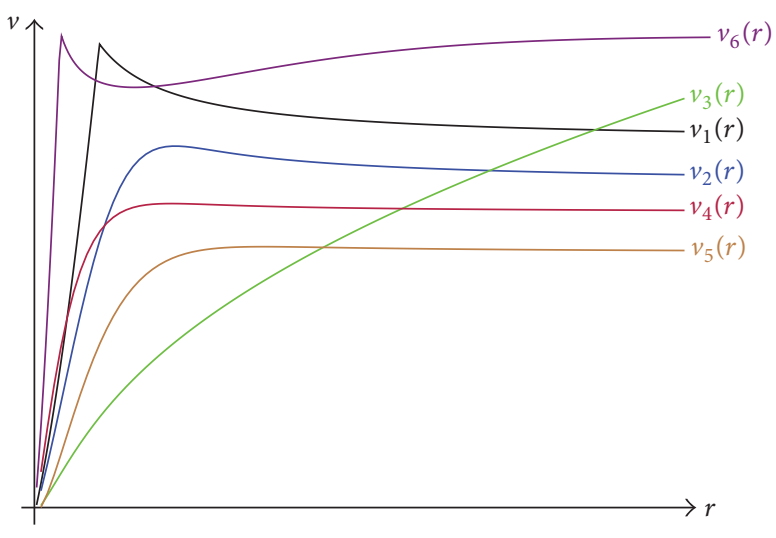

FIGURE 16

Furthermore, $\vec{F}$ is then only equivariant with respect to a subgroup of the central collineations because we must set $\vec{k} \equiv \sigma \vec{r}_{0}^{\prime}$. This is the result of (1) the central symmetry of $\vec{F}$ and (2) the Newtonian physics framework with the time and space splitting. Also, it can be noticed that in this Newtonian context, the terms such as $\beta t+\mu r_{0}$ in the expression of $\vec{F}$ may sound like a Minkowski inner product and could be the expression of a retarded Newtonian force as there exist retarded fields in electromagnetism.

In addition, we assume that the centripetal acceleration is a projective object and that it is modified in the same way as the Newton's law of gravitation: $v^{2} / r_{0} \rightarrow\left(\alpha+\beta t+\mu r_{0}\right) v^{2} / r_{0}$.

Then, if we modify the Newton's law of gravitation with a homography as indicated above preserving the mass distribution $\rho(r)$ to see the relative change between $\rho(r)$ and the radius $r$, then we can deduce the following rotational velocity field:

$$
\begin{aligned}
v(r) \equiv((1+a t+b r) & \left.\frac{M(r)}{r}\right)^{1 / 2} \\
& \text { where } M(r) \equiv \int_{0}^{r} u^{k} \rho(u) d u .
\end{aligned}
$$

Then, whenever $t=1$ and for different mass distributions $\rho$, we obtain the following qualitative curves if we consider $k=2$ for spherical distributions of mass in (36) (Figure 16 and Table 12).

Then, we see that the curves have a resemblance to the observed data. This suggests more exhaustive studies of the relations between galactic mass densities and rotational velocity fields according to relations (36) with varying exponent $k$. Moreover, the modified force $\vec{F}$ in (32) depends on the time $t$ which could be related to a notion of cosmological expansion, a relation which could be also studied in future works.

Finally, a last question arises from these projective aspects: what could be the vanishing points in such spacetime manifolds modeled locally by four-dimensional projective spaces? These vanishing points are at infinity in a projective space of dimension 3 , and then, they appear to be points of congruence of timelike worldlines not necessarily crossing in 
TABLE 12

\begin{tabular}{|c|c|c|c|}
\hline Rotational velocity & Mass distribution & $a$ & $b$ \\
\hline$v_{1}$ & $\rho_{1}(r)= \begin{cases}3 & \text { if } r \leq 1 \\
0 & \text { if } r \geq 1\end{cases}$ & -0.7 & 0.5 \\
\hline$v_{2}$ & $\rho_{2}(r)=e^{-r^{2}}$ (Gaussian) & -0.7 & 0.5 \\
\hline$v_{3}$ & $\rho_{3}(r)=\frac{1}{1+2 r^{2}}$ (Lorentzian) & -0.7 & 0.5 \\
\hline$v_{4}$ & $\rho_{4}(r)=e^{-r}$ (Exponential) & -0.7 & 0.5 \\
\hline$v_{5}$ & $\rho_{5}(r)=r e^{-r}$ & -0.7 & 0.5 \\
\hline$v_{6}$ & $\rho_{6}(r)=10000 \rho_{1}(10 r)+\rho_{5}(r)$ & -0.7 & 5 \\
\hline
\end{tabular}

the four-dimensional spacetime. Hence, could this produce a sort of Big-Bang effect?

\section{Conflicts of Interest}

The author declares that there are no conflicts of interest regarding the publication of this article.

\section{Acknowledgments}

The author would like to thank Professor Ralf Hofmann from Karlsruhe Institute of Technology (Karlsruhe, Germany) and Doctor Thierry Grandou from Institut de Physique de Nice (Valbonne, France) for their invitation to participate at the "5th Winter Workshop on Non-Perturbative Quantum Field Theory" which was held at Sophia Antipolis (22-24 March 2017, France) to present the principles of relativistic localizing systems.

\section{Endnotes}

1. Roughly speaking, let $x$ and $y$ be two events in spacetime. Then, (1) $x \prec y$ means that $y$ is in the future null cone of $x$ or in its interior, (2) $x \ll y$ means that $y$ is in the interior of the future null cone of $x$, and (3) $x \rightarrow y$ means that $x$ and $y$ are joined by a null geodesic starting from $x$ to $y$. The relation of order $\rightarrow$ is reflexive and it is also called the horismotic relation (see, e.g., [15, p. R9]).

2. In [16]: (i) Condition (V): $x \ll y \Rightarrow x \prec y$. (ii) Condition (VII): $x \rightarrow y \Leftrightarrow x<y$ and $x \ll y$.

3. Lemma 1-1 [16]: Let $x, y$, and $z$ be points in a causal space. If $x \prec y \prec z$ and $x \rightarrow z$ then $x \rightarrow y \rightarrow z$.

4. The ancillary emitter $\mathcal{S}$ can also be considered as the "soldering" emitter.

5. We can notice that projective Cartan connections differ from Ehresmann connections which are projector fields (in principal bundles) but projective Cartan connections are not; the word "projective" does not refer to a projection in a vector space but to the projective geometry/frames. Also, these two connections differ from the notion of Cartan connection in the sense of Ehresmann which is associated with the definition of the soldering map.

\section{References}

[1] J. L. Rubin, "Relativistic pentametric coordinates from relativistic localizing systems and the projective geometry of the spacetime manifold," Electronic Journal of Theoretical Physics, vol. 12, no. 32, pp. 83-112, 2015.

[2] B. Coll, "Elements for a theory of relativistic coordinate systems: formal and physical aspects," in Proceedings of the XXIII Spanish Relativity Meeting (EREs '00), J.-F. Pascual-Sánchez, L. Flora, A. S. Miguel, and F. Vicente, Eds., pp. 53-65, World Scientific Publishing Company, Incorporated, Valladolid, Spain, September 2000.

[3] T. B. Bahder, "Navigation in curved space-time," American Journal of Physics, vol. 69, no. 3, pp. 315-321, 2001.

[4] M. Blagojević, J. Garecki, F. W. Hehl, and Y. N. Obukhov, "Real null coframes in general relativity and GPS type coordinates," Physical Review, vol. 65, no. 4, Article ID 044018, 2002.

[5] C. Rovelli, "GPS observables in general relativity," Physical Review, vol. 65, no. 4, Article ID 044017, 2002.

[6] B. Coll, A principal positioning system for the Earth, in Journées 2002 - systèmes de référence spatio-temporels. Astrometry from ground and from space, (N. Capitaine and M. Stavinschi, eds.), vol. 14, pp. 34-38, 2003.

[7] B. Coll, J. J. Ferrando, and J. A. Morales, "Two-dimensional approach to relativistic positioning systems," Physical Review D, vol. 73, no. 8, Article ID 084017, 2006.

[8] B. Coll, "Relativistic positioning systems," AIP Conference Proceedings, vol. 841, no. 1, pp. 277-284, 2006.

[9] B. Coll, "Relativistic positioning systems," in Proceedings of the Workshop on Relativistic Positioning Systems and their Scientific Applications, ESA Advanced Concepts Team and the University of Ljubljana, Ljubljana, Slovenia, September 2012.

[10] J. Ehlers, F. A. E. Pirani, and A. Schild, "The geometry of free fall and light propagation," in General Relativity, Papers in Honour of J. L. Synge, L. O'Raifeartaigh, Ed., pp. 63-84, Clarendon Press, Oxford, UK, 1972.

[11] N. M. Woodhouse, "The differentiable and causal structures of space-time," Journal of Mathematical Physics, vol. 14, pp. 493501, 1973.

[12] S. W. Hawking, A. R. King, and P. J. McCarthy, "A new topology for curved space-time which incorporates the causal, differential, and conformal structures," Journal of Mathematical Physics, vol. 17, no. 2, pp. 174-181, 1976.

[13] D. B. Malament, "The class of continuous timelike curves determines the topology of spacetime," Journal of Mathematical Physics, vol. 18, no. 7, pp. 1399-1404, 1977. 
[14] H. Seifert and W. Threlfall, Seifert and Threlfall: A Textbook of Topology, vol. 89 of Pure and Applied Mathematics, Academic Press, London, UK, 1980.

[15] A. García-Parrado and J. M. Senovilla, "Causal structures and causal boundaries," Classical and Quantum Gravity, vol. 22, no. 9, pp. R1-R84, 2005.

[16] E. H. Kronheimer and R. Penrose, "On the structure of causal spaces," Mathematical Proceedings of the Cambridge Philosophical Society, vol. 63, no. 2, pp. 481-501, 1967.

[17] C. Ehresmann, Les connexions infinitésimales dans un espace fibré différentiable. Centre Belge Rech. Math., Colloque de Topologie, Bruxelles, 5th to 8th june 1950, 29-55, 1951.

[18] B. Famaey and S. S. McGaugh, "Modified Newtonian dynamics (MOND): observational phenomenology and relativistic extensions," Living Reviews in Relativity, vol. 15, no. 1, article 10, 2012.

[19] É. Cartan, The Theory of Spinors, Dover Publications, New York, NY, USA, 1981. 

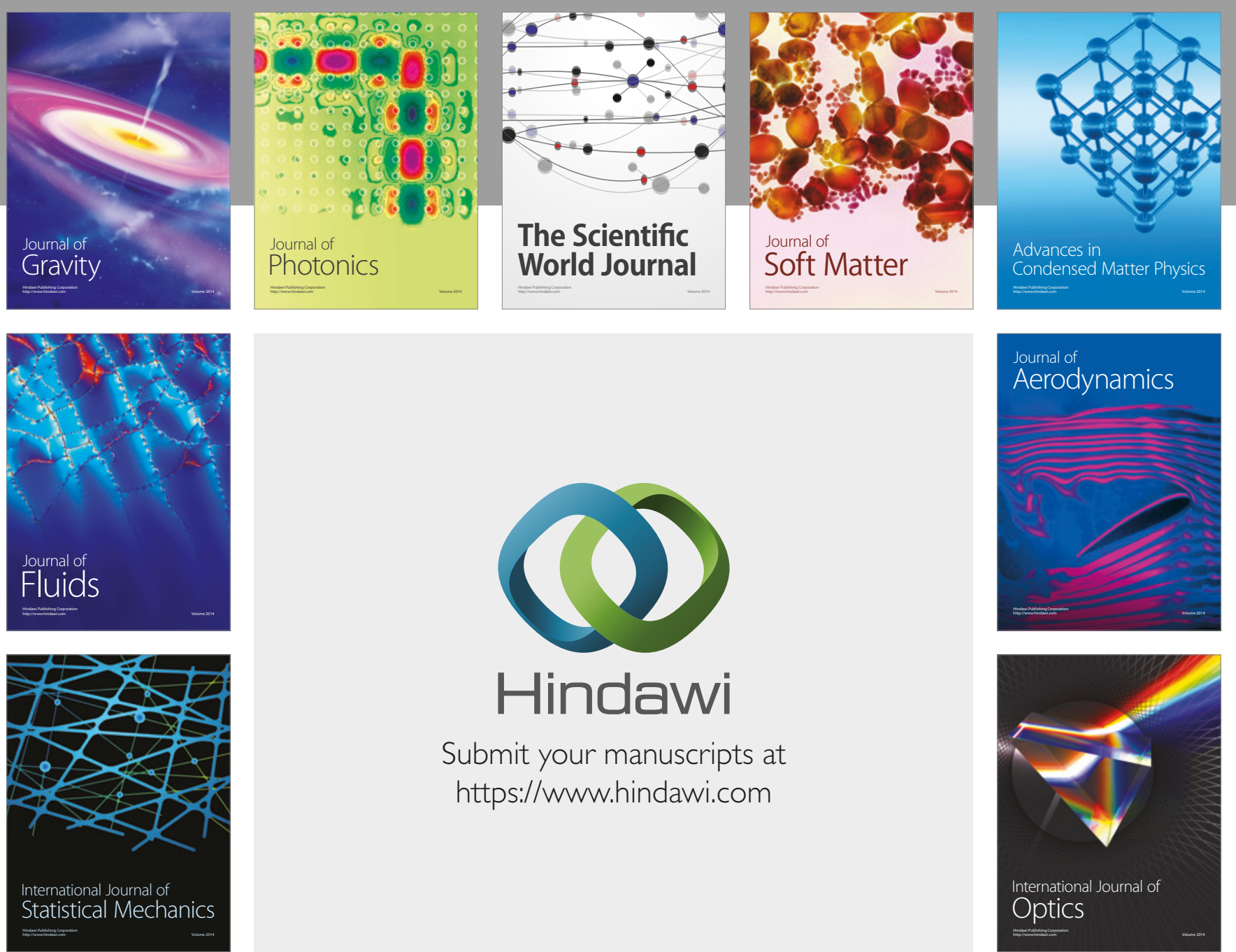

Submit your manuscripts at

https://www.hindawi.com
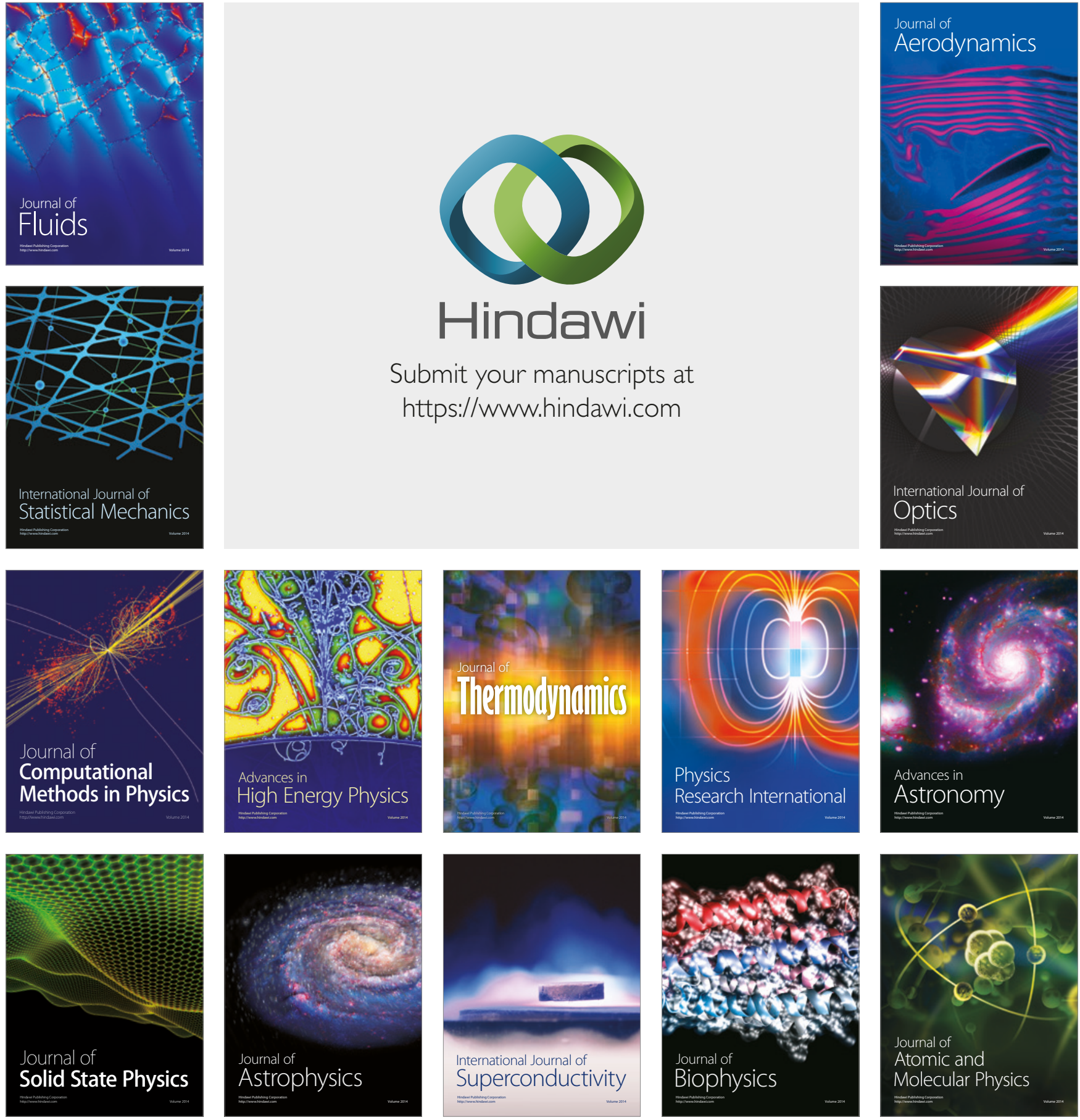